\title{
Experimental investigation of upstream flame propagation during boundary layer flashback of swirl flames
}

\author{
Dominik Ebi, Noel T. Clemens \\ Department of Aerospace Engineering and Engineering Mechanics, The University of Texas at Austin, \\ Austin, TX, 78712 \\ $+1(512) 471-6773$ \\ $+1(512) 471-3788$ \\ clemens@mail.utexas.edu
}

Boundary layer flashback of swirling turbulent lean-premixed methane-hydrogen-air flames is investigated in a model combustor featuring a mixing tube with center body. The focus of our work is on improving the understanding of the flow-flame interaction during flashback. We combine high-speed chemiluminescence imaging, stereoscopic and tomographic particle image velocimetry, and a three-dimensional flame front reconstruction technique to reveal the time-resolved, volumetric velocity field in the vicinity of the flame front during flashback. We find two different ways in which a flame front propagates upstream along the center body wall. The first mode concerns small-scale bulges counter-propagating into the approach flow, similar to channelflow flashback, but is found not to be a dominant propagation mechanism. Instead, flashback occurs primarily in the form of large-scale flame tongues swirling in the bulk flow direction as they propagate upstream. The approach flow is modified significantly in both cases, but the scale and nature of the resulting velocity fields differ fundamentally. A key characteristic of the approach flow found previously, both in channel and swirl flame flashback, is regions of negative axial velocity upstream of the flame front. We reveal, however, that in the case of swirl flames the region of negative axial velocity is the result of a primarily swirling motion ahead of the leading flame tongue in contrast to the reverse flow pockets ahead of small-scale bulges. The boundary layer neither separates nor does fluid recirculate in the negative axial velocity region upstream of the flame tongues. Instead, flame tongues impose a local blockage effect causing significant deflection of the approach flow, which results in a constant region of negative axial velocity for the leading side of the flame tongue to propagate into.

Keywords: Boundary layer flashback; Swirl flames; experimental; high-speed; Tomographic PIV; Turbulent flames 


\section{Introduction}

The successful design of future lean-premixed and fuel-flexible gas turbine combustors requires an improved fundamental understanding of flashback. Currently employed combustors designed to run on natural gas are challenged by the desire to use high-hydrogen content fuels owing to the fast kinetics, high diffusivity and low density of hydrogen.

Since research on flashback began with the first systematic study by Lewis and von Elbe [1,2], the focus has been on measuring flashback limits in (non-swirling) Bunsen-flame type burners for many years [3-7], including more recent studies carefully testing additional parameters such as confinement, wall temperature and pressure [8-13]. High-speed optical diagnostics and advanced simulation tools have been applied more recently to reveal new information about the flame propagation dynamics [14-17].

Three types of flashback can be distinguished: (i) flashback in the boundary layer of a (nonswirling) pipe or channel flow, (ii) flashback in the core of a swirling flow, and (iii) boundary layer flashback in a swirling flow. In all of these configurations a strong coupling between propagating flame front and approach flow has been found [14-19], which is in contrast to the originally proposed and still widely used critical gradient concept by Lewis and von Elbe. This concept assumes an isothermal flame and hence no effect of the heat release on the flow field. As a result of the coupling, flashback is possible in the non-trivial case of axial velocities exceeding the flame speed.

In recent studies, high-speed imaging and direct numerical simulation has shown that boundary layer flashback in channel flows is facilitated by small-scale flame bulges shaped convex towards the reactants, which intermittently form inside low-momentum streaks of the turbulent boundary layer $[16,17]$. These bulges cause local pockets of reverse flow reaching above the quenching distance, which are associated with a local pressure peak at the tip of the convex bulges. The formation, counter-propagation and break-up process of theses bulges leads to a net upstream propagation of the flame brush and hence flashback. A model to predict the flame shape and propagation speed has been developed recently [20].

Swirl flows are typically employed in practical combustors for enhanced mixing and flame anchoring purposes. The dynamics and stability of swirl combustors has been studied extensively [21]. Combustion instabilities may initiate a flashback process by providing, for instance, a momentary local low-momentum region or equivalence ratio stratification, which allows a portion of the flame to penetrate into the mixing tube. The danger for the combustor hardware lies in the sustained upstream flame propagation and flame anchoring inside the premix section [22]. 
Upstream flame propagation may occur in the core of a swirling flow, e.g. in combustors without a central fuel tube [23-30]. Flashback in such configurations is related to flame propagation along a vortex axis, and this mode of flame propagation has been studied extensively in laminar flows as summarized by Ishizuka [31]. A number of models have been developed aimed at predicting the significant increase in flame propagation speed with an increase in angular velocity and density ratio. Some of these concepts have been transferred to explain flashback along the mixing tube axis in swirl combustors. Flashback has been found to occur in form of a vortex-breakdown bubble, identified experimentally based on a region of negative axial velocity in an on-axis plane [14,19], which is continuously shifted upstream. The mechanism, termed combustion-induced vortex breakdown (CIVB) [23], points at the production of negative azimuthal vorticity due to baroclinic torque at the flame tip in analogy to the model by Ashurst [32], which induces a negative axial velocity on the tube axis and hence facilitates flashback [14,33,34].

The third configuration concerns flashback of swirl flames along a wall, which by geometry combines aspects of boundary layer flashback and flashback along a vortex axis. In swirl combustors featuring a mixing tube with center body, flashback typically occurs along the center body wall [15,35-38]; however, boundary layer flashback has also been observed along the mixing tube outerwall in hydrogen-air swirl flames [39]. Flashback along the center body wall has been attributed to CIVB based on the finding of regions of negative axial velocity ahead of the flame tip, identified as boundary layer separation, which pulls the flame upstream [15]. A model based on the modification of the axial pressure gradient along the center body wall due to the low-density burnt gas has been proposed [15]. Subsequent measurements of the static pressure on the center body wall during flashback revealed a pressure increase in the burnt gas, which supports this model [38].

Despite significant progress, the driving mechanism for flashback in swirl flames is not yet fully understood [15]. In particular, it remains unclear whether swirl-flame boundary-layer flashback is dominated by the mechanism driving flashback in non-swirling channel flows or that governing flashback in the core of swirling flows [38]. Furthermore, it is still unknown how the approach flow is modified ahead of the flame front in swirl flames as time-resolved measurements during the upstream flame propagation have so far focused on the axial and radial velocity fields only. Neither the azimuthal velocity component, which plays an important role in the models predicting flame propagation along the vortex axis, nor the full volumetric velocity field, have previously been measured with temporal coherence during flashback. We address the physics of flame propagation in swirling wall flashback by measuring in detail the modified approach flow, including the timeresolved volumetric velocity field, in the vicinity of leading flame fronts and study the resulting upstream flame propagation. 


\section{Experimental Setup}

\subsection{Swirl combustor}

The swirl combustor features an axial swirler with attached bluff body (center body) as shown in Fig. 1 allowing the investigation of boundary layer flashback of swirl flames. The burner is operated in fully-premixed mode in which the reactants are mixed before passing through the swirler. The fuelair-mixture is supplied through the four symmetrically arranged air-supply tubes. A combination of honeycomb section and wire-mesh elements inside the plenum ensured a clean inflow to the mixing tube. The single-axial swirler consisted of eight vanes and the vane trailing-edges were at an angle of $60^{\circ}$ relative to the tube axis. The swirl number is approximately $S \approx 0.9$ based on a numerical simulation of the flow field and is calculated as the ratio of axial to circumferential momentum flux based on time and space averaged radial profiles in a plane $10 \mathrm{~mm}$ upstream of the mixing tube exit. The hub diameter of the swirler is $25.4 \mathrm{~mm}$. A stainless-steel center body, of equal diameter to the swirler hub, was attached to the swirler, ending flush with the end of the mixing tube. The mixing tube was made of fused silica with high optical homogeneity (no lengthwise striations due to the manufacturing process) and had an inner diameter of $52 \mathrm{~mm}$ and a length of $150 \mathrm{~mm}$. The combustion section was directly downstream of the mixing tube and was composed of a quartz tube with an inner diameter of $100 \mathrm{~mm}$ and length of $150 \mathrm{~mm}$.

Experiments are conducted at atmospheric pressure. Air and fuel are supplied to the combustor at room temperature. Methane-air and hydrogen-methane-air mixtures are used as a fuel. Fast mass flow controllers (ALICAT MCR), controlled with LabVIEW, regulated the air and fuel mass flow rates. Flashback experiments started with a stable flame in the combustion chamber. The sudden expansion at the exit of the mixing tube caused vortex breakdown, which, together with the wake of the center body, led to a region of low axial velocity in the core of the combustion chamber. This low velocity region held the conically-shaped flame in place downstream of the center body. The distance between center body and flame base under stable conditions was about $10 \mathrm{~mm}$, depending on flow rate and equivalence ratio. Flashback was then initiated by a sudden increase in fuel mass flow to a predefined value. The change in fuel flow rate was fast enough that the flame propagated into constant equivalence ratio conditions in the mixing tube, which was tested by monitoring the increase in flame luminescence correlated with the arrival of the richer fuel-air-mixture.

The time from ignition to flashback was typically about 10 seconds to keep the thermal load on the combustor small. The center body was heated by both the incident laser sheet and the presence of the flame. Thermocouples were mounted inside the center body to monitor its temperature. The short time that the heat load was experienced by the center body led to a wall temperature of less than 
$100^{\circ} \mathrm{C}$ for all runs. Varying center body temperatures below $100^{\circ} \mathrm{C}$ have been found to have neither a noticeable effect on global flame speeds nor on the flame propagation direction. Any conclusions drawn in this work are hence for wall temperatures below $100^{\circ} \mathrm{C}$.

Quantitative results in this work are based on detailed measurements of ten methane-air flashbacks at an equivalence ratio of $\phi=0.8\left(s_{L}=0.26 \mathrm{~m} / \mathrm{s}\right)$ and five hydrogen-methane-air flashbacks with $95 \%$ hydrogen by volume and matched laminar flame speed $(\phi=0.4)$ at a Reynolds number of $\operatorname{Re}_{\mathrm{h}}=4,000$, based on the streamwise centerline velocity in the mixing tube annulus and the height $h$ of the annular gap. Measurements of an additional three methane-air flashbacks have been conducted at twice the flow rate $\left(\operatorname{Re}_{\mathrm{h}}=8,000\right)$ and $\phi=1.0$. Even though the number of investigated flashbacks is limited due to the transient nature of the problem, all findings reported in this work are repeatedly and consistently observed without any contradicting cases. All conclusions are furthermore supported by tens of additional investigated flashbacks leading up to this work, which were studied using similar experimental setups and moderately different conditions (flow rates, equivalence ratios, hydrogen contents).

\subsection{Diagnostics setup}

To study the coupling between flame propagation and approach flow high-speed laser Mie scattering from an aerosol seeded into the gas stream was used both for high-speed particle image velocimetry (PIV) and time-resolved flamefront imaging. The planar three-component and the volumetric velocity fields are measured by means of stereoscopic PIV and tomographic PIV, respectively. The planar and 3D flame topography are detected based on olive oil droplets, which vaporize at about $555 \mathrm{~K} \mathrm{[40]} \mathrm{and} \mathrm{hence} \mathrm{provide} \mathrm{a} \mathrm{marker} \mathrm{for} \mathrm{the} \mathrm{preheat} \mathrm{region} \mathrm{of} \mathrm{the} \mathrm{flame}$ subsequently referred to as flame front in this work. Employing the evaporation of oil droplets as a marker is a common technique [41-51] suited for the current study where the approximate flame front location in the region of the flame base is of interest. A marker capable of distinguishing a flame front from an interface between cold reactants and strongly pre-heated or burnt gas regions is not needed.

\subsubsection{Planar diagnostics}

Olive oil droplets of approximately $1 \mu \mathrm{m}$ in diameter were seeded into the flow and illuminated with two high-repetition rate diode-pumped, frequency-double $527 \mathrm{~nm} \mathrm{Nd:YLF} \mathrm{laser} \mathrm{(Coherent}$ Evolution-90) operated with about $10 \mathrm{~mJ}$ per pulse at a repetition rate of $4 \mathrm{kHz}$. A long focal length spherical lens and a set of cylindrical lenses were used to form a collimated $20 \mathrm{~mm}$ wide laser sheet with a thickness of about $1 \mathrm{~mm}$. The laser sheet entered the mixing tube from the top in the $r$-z-plane. The sheet was aligned with the mixing tube axis as shown in Fig. 2. Two high-speed CMOS cameras (FASTCAM-ultima APX) were operated in forward scattering mode at a framing rate of $8 \mathrm{kHz}$ and a 
resolution of 256 x $512 \mathrm{pix}^{2}$. The cameras were fitted with Scheimpflug adapters and Nikkor $105 \mathrm{~mm}$ lenses operated with an $f / 5.6$ aperture. The angle between cameras and light-sheet normal was $30^{\circ}$. The field-of-view is $13.5 \times 27 \mathrm{~mm}^{2}$ with a pixel resolution of about $50 \mu \mathrm{m}$. The top edge of the fieldof-view is located at $z=-42 \mathrm{~mm}$, meaning $42 \mathrm{~mm}$ upstream of the mixing tube exit plane. In the current experimental setup, the center body can be removed for calibration purposes. A calibration target was traversed through the laser sheet in order to obtain the mapping function between pixel location and physical space, which was used to dewarp the Mie scattering images.

To enable measurements close to the center body, the light sheet was brought in from the top and grazed along the wall, which is a frequently employed strategy for near-wall measurements as it significantly reduces reflections off the center body. However, an additional difficulty arises in measurements where a flame propagates along the wall as is the case in boundary layer flashback studies: The light is refracted towards the wall due to the density gradients associated with the presence of the flame. To mitigate the occurrence of bright spots on the wall falsifying the crosscorrelation in interrogation regions close to the wall, the cameras were rotated into a stereoscopic setup in the $z-\theta$-plane (as opposed to the $r-\theta$-plane) as shown in Fig. 2 such that the line-of-sight was parallel to the tangent on the center body wall. With this setup, bright spots due to reflections were found to be limited to within one pixel from the wall restricting the necessary masking in that region and enabling valid velocity vectors to be computed close to the wall.

The three-component planar velocity field was measured at $4 \mathrm{kHz}$ and processed with the LaVision software $\mathrm{DaVis} 8.2$. The final interrogation window size was $16 \times 16 \mathrm{pix}^{2}$ with $75 \%$ overlap corresponding to $0.8 \times 0.8 \mathrm{~mm}^{2}(\sim$ spatial resolution) in physical space with a vector spacing of $0.2 \mathrm{~mm}$. The cross-correlation, performed over a circular interrogation window with Gaussian weighting, typically detected more than $95 \%$ of the vectors with a signal-to-noise ratio (ratio between first and second correlation peak) of 2 . The uncertainty in the planar velocity measurement is estimated in two ways. First, the mean uncertainty associated with the random error is computed based on the correlation statistics approach [52]. For the lower Reynolds number case, the mean uncertainty associated with the instantaneous radial and axial velocity field is $0.10 \mathrm{~m} / \mathrm{s}$ with a standard deviation of $0.02 \mathrm{~m} / \mathrm{s}$. The out-of-plane (azimuthal) velocity component has an uncertainty of $0.19 \mathrm{~m} / \mathrm{s}$ with a standard deviation of $0.04 \mathrm{~m} / \mathrm{s}$. Second, the planar three-component velocity field was measured with two independent sets of cameras in a similar configuration (even less favorable camera angles) to obtain a redundant solution [40]. The resulting mean deviation, which includes any bias error (e.g. due to calibration inaccuracies and beam steering effects) in addition to the random error, was between $0.05 \mathrm{~m} / \mathrm{s}$ and $0.13 \mathrm{~m} / \mathrm{s}$. The estimated uncertainties correspond to about $3 \%$ uncertainty in the coreflow of the in-plane components and about $5 \%$ for the azimuthal component. These relative uncertainties are comparable in the higher Reynolds number case. 
The flame front was determined based on vaporized olive oil droplets for every other frame in the movie sequence in order to match the temporal resolution of the velocity field $(4 \mathrm{kHz})$. The automated flame front extraction is based on a threshold intensity in $8 \times 8$ pix $^{2}$ probe windows with $75 \%$ overlap. A series of morphological operations was implemented to eliminate spurious patches both within and outside the area occupied by the flame. The resulting flame front was filtered to avoid artificial high-frequency wrinkles due to the discrete nature of the particle pattern. A sample particle image with flame and extracted flame front is shown in Fig. 3. The final flame front plotted together with the measured velocity field in subsequent sections is the mean flame front obtained from the two available flame front locations (one from each PIV camera). The uncertainty associated with the automated detection of the flame front is about $0.1 \mathrm{~mm}$ based on the two independent solutions available and hence sufficiently small for any conclusion drawn in this work based on the approximate location of the preheat zone.

The time-resolved chemiluminescence of the flame was simultaneously recorded with an intensified high-speed CMOS camera (FASTCAM-ultima APX-i2). The flame was imaged at $4 \mathrm{kHz}$, with a resolution of $512 \times 512$ pix $^{2}$. The camera was fitted with a Nikkor $105 \mathrm{~mm}$ lens with an $f / 2.8$ aperture. The shutter and gain gate was timed such that the luminescence was recorded between the two laser pulses for the Mie scattering images for best agreement between chemiluminescence images and velocity field data. The camera was setup such that the luminescence was viewed perpendicular to the laser sheet as shown in Fig. 2. Hence, information on how the flame propagates in and out of the laser sheet was available, which enabled an unambiguous interpretation of the planar velocity field and its location relative to a flame tongue.

\subsubsection{Volumetric diagnostics}

In a recent study we presented an approach capable of detecting the three-dimensional flame topography based on the reconstructed volumetric droplet field and simultaneously measure the velocity in the vicinity of the flame by means of tomographic PIV. The technique and its validation are described in detail in [40]. Only the main features are summarized here. The volumetric and planar measurements are not conducted simultaneously in this work as the same hardware is used to investigate flashback in different fields-of-view.

Micrometer sized olive oil droplets were illuminated with a high-repetition rate laser (Coherent Evolution-90) providing about $13 \mathrm{~mJ} /$ pulse of $527 \mathrm{~nm}$ light at a rate of $5 \mathrm{kHz}$. A thick sheet was formed and retro-reflected to balance the scattering among all cameras as shown in Fig. 4. A homogenous illumination to within $10 \%$ of the peak laser fluence was achieved in a volume of $25 \times 25 \times 4.5 \mathrm{~mm}^{3}$, which was taken as the final measurement domain size. The measurement domain 
corresponds to a secant through the annulus with the back-side of the domain being offset from the center body wall by about $0.8 \mathrm{~mm}$ in order to prevent severe reflections off the center body.

The Mie scattering was recorded with four high-speed CMOS cameras (Photron FASTCAMultima APX) operated at $5 \mathrm{kHz}$ at a resolution of $512 \times 512$ pix $^{2}$ with about $60 \mu \mathrm{m} /$ pix. The cameras were fitted with Scheimpflug adapters and Nikkor $105 \mathrm{~mm}$ lenses set to an aperture of $\mathrm{f} / 8$ providing a sufficiently high depth-of-field. The cameras were oriented in a diagonal cross-like arrangement with a total camera angle of about $60^{\circ}$, which is optimal for the tomographic reconstruction [53]. The cameras were calibrated by removing the center body and traversing a dot target through the volumeof-interest. The calibration for the tomographic reconstruction was improved by applying the volume self-calibration routine [54]. The remaining disparity ranges from 0.08 pix in the core of the volume to 0.15 pix close to the curved fused silica mixing tube walls, which is sufficiently small for a good reconstruction $[53,54]$.

The tomographic reconstruction to obtain the volumetric voxel-based intensity distribution was performed using the fast MART (CSMART) algorithm (6 iterations) within the LaVision DaVis 8.2 software. The reconstructed particle field was improved by performing two iterations of MotionTracking-Enhancement (MTE) [55]. The reconstruction quality (ghost particle level) is assessed based on the reconstruction signal-to-noise ratio $\mathrm{SNR}_{\mathrm{R}}[53,56]$. The $\mathrm{SNR}_{\mathrm{R}}$ ranges from 1.7 to 2.3 for different runs, which is at the lower limit of the acceptable level and a direct result of the challenges associated with performing these measurements through curved glass windows and with a high background intensity due to the center body wall [40].

In analogy to the planar measurements, the flame surface is defined as the interface between regions with droplets (unburnt gas) and void regions (burnt gas). A Matlab routine was developed to detect void regions and extract the flame surface as described in detail in [40]. The uncertainty associated with the three-dimensional flame topography is $0.4 \mathrm{~mm}$, which was assessed by comparing the flame-surface location to four independent simultaneously-acquired planar flame fronts at different locations within the volume [40].

The remaining PIV processing to obtain the volumetric velocity field was performed in $\mathrm{LaVision} \mathrm{DaVis}$ 8.2. Regions of burnt gas were algorithmically masked in the reconstructed particle field based on the detected void regions. The cross-correlation was performed with a final interrogation volume size of 32 voxels $^{3}$ with a $75 \%$ overlap corresponding to $1.92 \mathrm{~mm}^{3}$ with a vector spacing of $0.48 \mathrm{~mm}$. Spurious vectors were detected by the universal outlier detection scheme in a $5 \mathrm{x}$ $5 \times 5$ vector neighborhood in space and a 3-vector neighborhood in time [57]. No spatial smoothing was applied to the final vector field. The uncertainty in the measured volumetric velocity is assessed by comparing the volumetric velocity field with a redundant set of simultaneously measured planar, 
three-component velocity fields in different slices through the volume. Absolute mean errors between $0.16 \mathrm{~m} / \mathrm{s}$ and $0.21 \mathrm{~m} / \mathrm{s}$ were found for all three velocity components [40].

\section{Results}

\subsection{Non-reacting swirl flow in annular mixing tube}

Flashback experiments presented in this work were conducted at atmospheric pressure and Reynolds numbers of about $\operatorname{Re}_{\mathrm{h}}=4,000$ and 8,000, respectively. The centerline velocity fluctuations are about $0.5 \mathrm{~m} / \mathrm{s}$ and $1.0 \mathrm{~m} / \mathrm{s}$ for the lower and higher $\mathrm{Re}_{\mathrm{h}}$, respectively, corresponding to a turbulence intensity of about $10 \%$ in each case. The non-reacting radial velocity profiles for each Reynolds number are shown in Fig. 5. The left axis corresponds to the location of the center body wall $(r=0 \mathrm{~mm})$; the right axis to the location of the mixing tube wall. Valid velocity data was not obtained close to the outer (mixing tube) wall due to severe image distortion. The swirl flow generated in this particular model combustor is characterized by axial velocities (blue lines) that increase towards the mixing tube wall before falling off in the outer boundary layer (not seen here). The azimuthal velocity (green) peaks at about $r=3 \mathrm{~mm}$. The peak value and subsequent fall off is prominent for the higher $\mathrm{Re}_{\mathrm{h}}$ case. The axial and azimuthal profiles translate into a local swirl angle, defined as the angle between mixing tube axis and streamwise velocity vector, plotted in red. The swirl angle is higher close to the center body wall and decreases towards the mixing tube wall. Hence, the azimuthal momentum relative to the axial momentum is higher close to the center body wall. This effect is stronger in the higher bulk flow velocity case where the swirl angle is about $65^{\circ}$ close to the inner wall in comparison to about $55^{\circ}$ for the lower velocity case. The radial variation of the swirl angle and the variation of swirl angle with a change in bulk flow velocity but constant swirler geometry may suggest that a fixed global swirl number based on the swirler geometry is not suited for scaling quantities like a global flame propagation speed, in which case the flame predominately experiences the swirl close to the center body wall in this configuration.

\subsection{Qualitative flame shape and propagation direction}

The upstream flame propagation during a flashback event is first discussed qualitatively based on high-speed chemiluminescence movies. The luminescence of a $\mathrm{CH}_{4}$-air flame undergoing flashback at $\operatorname{Re}_{\mathrm{h}}=4,000$ and $\phi=0.7$ is shown in Fig. 6 at selected time steps taken from a movie sequence recorded at $2 \mathrm{kHz}$. The time $t_{0}$ corresponds to the instant when an increase in flame luminescence is first detected visually. This increase in luminescence immediately precedes flashback. The flow swirls in the counter-clockwise direction as indicated by the red arrows. The flame tongue leading the flashback is convected with the bulk flow in the azimuthal direction as it propagates upstream along 
the center body wall (indicated by the blue arrows in the first two time steps). We use the term "flame tongue" rather than "flame front" to emphasize that the leading part of the flame is a protuberance or large bulge rather than an azimuthally-uniform flame surface. At $t_{0}+257 \mathrm{~ms}$, for instance, the flame tongue is on the back side of the center body but no portion of the flame propagates upstream on the front side. The characteristic shape of the leading flame tongues and their swirling motion in the direction of the bulk flow as they propagate upstream holds for all flames and flow rates investigated as part of this work (tens of individual flashback events). Presented chemiluminescence image sequences during flashback in a similar configuration appear to support these observations $[35,38]$.

Additional large flame tongues may form on either side of the leading flame tongue as is seen for example at $t_{0}+355 \mathrm{~ms}$ where the leading flame tongue is on the right side of the center body and a secondary flame tongue has formed and swirls into the field-of-view on the left (circled with a yellow line). However, only one large flame tongue is found to prevail during flashback events under most conditions; either the originally leading one continues to lead the flashback or a newly formed one is taking over. Additional flame tongues are typically washed downstream and only coexist for longer periods of times if they form rather far away from the leading flame tongue in the azimuthal direction, which suggests that conditions are not favorable for upstream flame propagation immediately to the left or right of a leading flame tongue in agreement with the velocity field measurements discussed in Section 3.4 .

The shape and propagation direction of flame tongues is investigated in more detail for a $\mathrm{CH}_{4}-$ air flame (Fig. 7(a) and Video 1(a)) and an $\mathrm{H}_{2} / \mathrm{CH}_{4}$-air flame (Fig. 7(b) and Video 1(b)). The field-ofview is shown in red in the last time step of Fig. 6 . We focus on a region about half way down the mixing tube to investigate the sustained upstream flame propagation in a region where the flow field is not affected by geometry-dependent mixing tube entry or exit effects. In Fig. 7(a) and (b), at time $t_{1}$, the leading flame tongue is swirling around the center body into the field-of-view as indicated by the green arrow. The nominal flow direction is indicated by the red arrow. The edges of the center body are outlined by the vertical grey lines. We again refer to the rather large portion of the flame that is leading the flashback as a flame tongue as indicated for both flames at time $t_{2}$. In contrast, we refer to smaller structures along the flame front as flame bulges. Such flame bulges tend to form on the trailing side of the flame tongue. Flame tongues and flame bulges, as defined in this work, may be differentiated quantitatively in terms of their size (width or radius of curvature), which differs by an order-of-magnitude. Flame tongues have a characteristic size on the order of the geometry of the mixing tube (diameter of the center body or $20-30 \mathrm{~mm}$ ) since they describe the asymmetry of the overall flame front along the circumference of the center body. Instead, flame bulges have a characteristic width on the order of 2 to $3 \mathrm{~mm}$ and are hence comparable in size with the measured integral scale of turbulent structures in the investigated flow. 
In the case of the methane flame ( $\phi=0.8$ ), flame bulges form occasionally with an example seen in Fig. 7(a) at time step $t_{3}$ and highlighted by the yellow dashed circle. This bulge attempts to propagate upstream in the negative streamwise direction as indicated by the yellow arrow and manages to momentarily resist the approach flow - it remains at a fixed location in space for about $3 \mathrm{~ms}$ - while the tip of the leading flame tongue continues to swirl upstream (green arrow). However, the bulge then breaks into two smaller bulges - likely due to the arrival of a faster moving flow structure - and is convected downstream as seen $6.25 \mathrm{~ms}$ later at time $t_{4}$. While the flame bulge fails to initiate sustained upstream flame propagation, the tip of the leading flame tongue continues to swirl upstream as indicated by the green arrows at time steps $t_{2}$ and $t_{3}$. By time step $t_{4}$ it is on the back side of the center body.

The $\mathrm{H}_{2} / \mathrm{CH}_{4}$-air flame (95\% hydrogen by volume, $\left.\phi=0.4\right)$ shown in Fig. $7(\mathrm{~b})$ has the same laminar flame speed but a significantly lower flame temperature as the previously discussed methane flame but features a more convoluted flame surface, which may be attributed to thermo-diffusive effects [59]. The overall shape of the flame during flashback, however, is again characterized by a large flame tongue leading the flashback as seen at times steps $t_{1}$ and $t_{2}$. The formation of small-scale bulges located on the trailing side of the leading flame tongue and aligned with the nominal streamwise direction are more frequently observed for high-hydrogen content flames compared to the previously discussed methane flames. Furthermore, the bulges are smaller in size and manage to not just resist the approach flow (freeze at a fixed location in space) but sometimes even counter propagate into the approach flow for short distances $(\sim 1 \mathrm{~mm})$, which may be attributed to a smaller quenching distance and an increase in flame speed at the tip of these convex shaped bulges owing to local enrichment effects of the deficient reactants. Based on the full movie sequence (Video 1(b)), the two bulges marked by the yellow circle at time step $t_{3}$ are being convected downstream whereas the bulge marked by the orange circle manages to counter propagate into the approach flow for a short time $(\sim 1 \mathrm{~ms})$ and distance. About $11 \mathrm{~ms}$ later (time step $t_{4}$ ), this same bulge is seen (again marked by an orange circle), but now broken into two separate bulges and again washed downstream as indicated by the small orange arrow. All the while, the tip of the flame tongue leading the flashback continues to swirl upstream; it is already on the right side of the center body at $t_{3}$ (indicated by the green arrow) and on the back side below the bottom edge of the field-of-view at $t_{4}$.

The formation and break-up of the small-scale bulges on the trailing side of the leading flame tongue, particularly for the $\mathrm{H}_{2} / \mathrm{CH}_{4}$-air case, agree well in size and occurrence with the observations reported by Eichler et. al [16] investigating flashback in a (non-swirling) channel flow. This is reasonable as the trailing sides of the swirling flame tongues observed in the current work are aligned approximately perpendicular to the streamlines of the approach flow (as will be discussed in Section 3.4.2., Fig. 11, and Section 3.4.3., Fig. 13) just like in a channel flashback where the flame brush is 
oriented perpendicular to the flow. However, the contribution of these flame bulges to facilitate the overall flashback is different. In a channel boundary layer flashback, a combination of parameters likely to include flame speed, quenching distance and density ratio (the relative importance of each of these factors is not yet fully understood) has to be above a particular threshold such that the formation, upstream propagation, break-up and downstream convection of these small-scale bulges leads to a net upstream propagation of the entire flame brush. The requirement for the flame to achieve overall flashback in a swirl flow is different, however. Instead of fighting the approach flow head-on in form of small-scale bulges, the flame is found to flashback in the form of large-scale flame tongues swirling upstream, which appears to be the path of least resistance for the flame even when flashback was triggered at an equivalence ratio significantly beyond the flashback limit.

In summary, we consistently observe two modes of upstream flame propagation during boundary layer flashback in swirl flows. The first mode consists of large-scale flame tongues swirling in the direction of the bulk flow as they propagate upstream. The second mode concerns small-scale flame bulges aligned with the streamwise direction, which - particularly for high-hydrogen-content flames - manage to resist the approach flow head-on and counter-propagate into the approach flow (negative streamwise direction) for short distances $(\sim 1 \mathrm{~mm})$ but do not provide net upstream flame propagation on their own. The first and second mode will be investigated in more detail in Sections 3.3 and 3.4, respectively, by discussing the velocity field in the vicinity of the flame front associated with each mode.

\subsection{Flame propagation associated with small-scale bulges}

We compare the velocity field upstream of small-scale bulges with non-swirling channel flashback results by Eichler et al. [16] and Gruber et al. [17] to provide evidence that the same mechanism facilities the upstream flame propagation associated with such bulges in our swirl flow as suggested qualitatively by the time-resolved chemiluminescence imaging discussed in Section 3.2. The chemiluminescence image sequence in Fig. 8 shows the formation of a small-scale bulge on the trailing side of a flame tongue during a $\mathrm{CH}_{4}$-air flame flashback $(\phi=0.8)$. Results from the volumetric velocity field measurement corresponding to an instant in time when the flame bulge resists the approach flow (the local displacement speed matches the flow velocity) is shown in Fig. 8(a). The three-dimensional flame topography based on our marker for the preheat zone is shown in grey. The axial velocity field is shown in a secant through the annulus, which is offset from the center body by about $0.8 \mathrm{~mm}$ at its closest point as described in Section 2.2.2. The white band separates positive from negative axial velocity with the region of negative axial velocity colored in blue. A reverse flow pocket, marked by the black solid line corresponding to zero streamwise velocity, exists in agreement with previous studies $[16,17]$. Three-dimensional streamlines are plotted and color coded by the $y$ - 
distance to indicate their location in the depth direction as indicated in Fig. 8(c), which is a bottom view of Fig. 8(a) highlighting again the location of the measurement volume in relation to the center body with the back side of the measurement domain slightly offset from the center body wall.

Flame bulges locally push the low-momentum near-wall fluid upstream in the negative streamwise direction, which leads to a stagnation point at the upstream end of the reverse flow pocket. The near-wall streamlines (green) diverge symmetrically to either side of the flame bulge. Streamlines farther offset from the wall (yellow and orange) still diverge but to a lesser extent. The agreement in the velocity field with the DNS results by Gruber et al. suggests that the propagation mechanism associated with these small-scale bulges is indeed the same. The DNS was further able to show that the reverse flow pockets are associated with a localized pressure peak ahead of the convex shaped bulges whereas the pressure drops downstream of the flame brush. The location where convex shaped bulges form was found to be correlated with the occurrence of low-momentum streaks [17] as previously suggested by Eichler et al. [16]. It appears that in our swirl flow the formation of smallscale bulges is also linked to the arrival of low-momentum fluid either due to low-momentum streaks in the boundary layer or due to wake effects originating from the swirler vanes. However, since we cannot measure the velocity along the circumference of the center body but only in a tangent or wallnormal plane, we cannot establish the correlation with certainty. As described before, small-scale bulges are found not to sustain in our swirl flow and are washed downstream shortly after their formation as shown in Fig. 8(b).

\subsection{Flame propagation associated with large flame tongues in swirl flows}

\subsubsection{Region of negative axial velocity}

Regions of negative axial velocity upstream of the leading flame front have first been observed by Heeger et al. [15] in a similar swirl burner configuration featuring a mixing tube with center body along which the flame propagates during flashback. The planar two-component velocity field in a radial-axial slice was measured in their work while simultaneously recording the flame luminescence from a point-of-view perpendicular to the planar measurement domain. Apparent upstream propagation without negative axial velocity was observed in about half of the recorded cases. The possibility that such cases correspond to instances when the laser sheet slices through the leading or trailing side of a flame tongue rather than the leading tip was suggested.

Our measurements consistently show that upstream propagation without a negative axial velocity region is an artifact associated with a planar measurement in a 3D flow in agreement with the suggestion by Heeger et al. As an example, a few selected time steps of a methane-air flashback $(\phi=0.8)$ movie are shown in Fig. 9. The luminescence is shown in the top row with arrows indicating 
the nominal flow direction and the flame propagation direction as well as the PIV measurement domain (green vertical line). The corresponding velocity fields are shown in the bottom row with the grey vertical bar on the left indicating the location of the center body wall and the red line indicating the flame front (based on the employed marker for the preheat zone). The planar velocity field and flame front location measurement by itself would suggest that flashback is characterized by an initial flame propagation away from the wall (Fig. 9(b)) followed by flame propagation along the wall without a region of negative axial velocity upstream of the flame base (Fig. 9(c)) and finally upstream flame propagation with a region of negative axial velocity (Fig. 9(d)). In contrast, the flame luminescence reveals that the apparent upstream propagation is instead a swirling motion as previously discussed where the flame is behind the center body in Fig. 9(a) and the measurement slices through a downstream portion of the flame in Fig. 9(b) and (c). Only Fig. 9(d) corresponds to a time step when the flame tip is in the planar field-of-view. We confirmed all findings just discussed based on planar measurements with high-speed volumetric measurements to be discussed in the subsequent sections. Studying certain aspects of the three-dimensional flame-flow interaction during swirl flame flashback is therefore possible if some means to unambiguously interpret the planar data is available such as luminescence imaging from a perpendicular point-of-view in this case. True upstream propagation of the leading flame tip is found always to be associated with a region of negative axial velocity ahead of the flame tip for all conditions and all runs conducted as part of our work.

The region of negative axial velocity upstream of the leading flame tongue is found to exist not only immediately at the flame tip but also on its leading side corresponding to the fluid region the flame is propagating into as shown in Fig. 10(a). On the leading side of the flame tongue negative axial velocity is observed in the near vicinity of the flame front only. In contrast, the region of negative axial velocity reaches far upstream in the vicinity of the flame tip as shown in Fig. 10(b). The length-scale of this negative axial velocity region is about 5 to $10 \mathrm{~mm}$ in extent based on observations from ten flashback events. This observation of the extent of negative axial velocity holds even for more turbulent conditions (twice the bulk flow velocity corresponding to $\operatorname{Re}_{\mathrm{h}}=8,000$ ).

As discussed in Section 3.2., the investigated $\mathrm{H}_{2} / \mathrm{CH}_{4}$-air flames $(95 \%$ hydrogen by volume, $\phi=0.4$ ) are found to be more convoluted as a result of small-scale bulges, which form more frequently compared to the methane-air flames. However, focusing on the similarities in this work, the upstream flame propagation again occurs in form of larger flame tongues. The velocity measurements show regions of negative axial velocity upstream of the leading flame tongue, which compare well with the findings in methane-air flames. The observed axial extent of the negative axial velocity region appear to be smaller (about $5 \mathrm{~mm}$ ), but a wider range of conditions would need to be tested before more general conclusions can be drawn. 


\subsubsection{Three-dimensional velocity field in the vicinity of leading flame tongues}

The volumetric velocity field in the vicinity of flame tongues, which dominate boundary layer flashback in the investigated swirl flames, is shown in Fig. 11. The axial velocity field is seen in a secant through the annulus offset from the center body by about $0.8 \mathrm{~mm}$ as in Fig. 8 . The blue colored region bounded by the white band shows a region of negative axial velocity. The color coding for the three-dimensional streamlines follows Fig. 8(c) indicating the $y$-distance from the center body. The chosen instant in time captures the tip of a larger flame tongue that is swirling with the bulk flow velocity in the counter-clockwise direction as it propagates upstream, which corresponds to the flame motion found to lead flashback in the investigated swirl flames. The velocity field is fundamentally different compared to the velocity field upstream of small-scale bulges as presented in Fig. 8. The region of negative axial velocity is merely the result of a rather large-scale deflection of streamlines as opposed to a localized reverse flow pocket, which is emphasized by the near-wall streamlines (green) deflected around the tip of the flame tongue without being reversed. The azimuthal velocity component remains large upstream of the flame tongue in contrast to the reverse flow pockets which are the results of both negative axial and negative azimuthal velocity. Note that we define "reverse flow" as a region with fluid that flows counter to the dominant upstream flow direction. In the case of a swirling flow, reverse flow propagates in the negative swirl direction. A moderate level of deflection of streamlines in the investigated swirl flow is sufficient to result in a region with a negative axial component, but without flow reversal.

The velocity field is shown in a perpendicular plane in Fig. 11(b), which corresponds to a radial-axial slice typically measured with a planar technique as presented previously in Fig. 9 and 10 . The tangent vectors suggest flow recirculation, which has been interpreted as boundary layer separation in previous works [15]. However, neither flow recirculation nor boundary layer separation occurs upstream of the preheat zone in this case as the volumetric measurements reveal. Furthermore, the location where the velocity vectors switch from a positive to a negative axial component at the upstream end of the negative axial velocity region is not a stagnation point but instead corresponds to purely swirling flow. A stagnation point may still exist inside the burnt gas, however.

The volumetric measurement readily provides the explanation for the difference in characteristic shape and axial extend of the negative axial velocity region between the leading side of the flame tongue and at the flame tip as observed previously in planar measurements shown in Fig. 10. Instead of diverging to either side of the flame tongue, the flow is deflected downwards ahead of the flame tongue as well as alongside the leading side of the flame tongue, hence providing a region of negative axial velocity for the flame to propagate into. 
Fluid farther out in the radial direction is only moderately deflected in the axial direction as the streamlines colored in red indicate (Fig. 11). Hence, a shear layer forms between the near-wall layer of fluid with a predominantly swirling motion and fluid away from the wall moving at a swirl angle closer to the nominal angle and hence with a significant axial component. The shear layer consists of negative azimuthal vorticity, which may also be measured with planar PIV in a radial-axial slice as shown in Fig. 12 and Video 2. The red line marks the location of the flame front. The grey vertical line along the left edge indicates the location of the center body wall. The shear layer is indicated by the dashed black line. Figure 12(a) corresponds to the time step shown in Fig. 10(a), at which the leading side of the flame tongue is in the field of view. Negative azimuthal vorticity is concentrated in the near vicinity of the flame front. The flame continues to swirl into the measurement plane, which is associated with shear appearing farther upstream of the flame. Figure 12(d) corresponds to the instant in time shown in Fig. 10(b) when the laser sheet cuts through the flame tip. At this time, a strong shear layer exists between the near-wall fluid with a negative axial velocity and fluid moving in the positive axial direction farther away from the wall.

The production of negative azimuthal vorticity at the flame tip due to baroclinic torque, which results from the axial density gradient across the flame tip and the radial pressure gradient due to the swirl flow, has been proposed as a mechanism enhancing the flame speed along a vortex axis [32]. The concept of vorticity produced by baroclinic torque and inducing a negative axial velocity has subsequently been identified as a mechanism driving flashback in the core of swirl flows associated with combustors not featuring a center body in the mixing tube [14,19,33]. Our measurements suggest that in boundary layer flashback of swirl flames the observed negative azimuthal vorticity present in the immediate vicinity of the flame tip is part of a larger coherent shear layer, which reaches far upstream into the cold approach flow and hence is not dominated by vorticity production due to baroclinic torque. The data hence suggest that the observed azimuthal vorticity is not the cause but an effect of the upstream flame propagation. The observed concentration of elevated azimuthal vorticity may be the result of already existing boundary layer vorticity, which is being reoriented and transported away from the wall by the modified flow field in the vicinity of the leading flame tip. Additional vorticity may possibly be produced at the center body wall due to a pressure gradient induced by the presence of the flame tongue.

\subsubsection{Region of influence of the local blockage effect}

As described in the previous section and highlighted in Fig. 11, flame tongues decelerate and deflect the approach flow. The effect flame tongues have on the approach flow may hence be described as a blockage effect due to the gas dilatation associated with the heat release. It is instructive to investigate where and how the velocity field is influenced at various points relative to a flame 
tongue. For that purpose time traces of axial velocity, azimuthal velocity and swirl angle (angle between the axial direction and the instantaneous, local streamwise flow direction) are evaluated at two points in space as shown in Fig. 13. Both points are located at the same axial location as marked by a white cross in the sequence of chemiluminescence images shown in Fig. 13(a), which provides the flame location in relation to the measurement points. The instantaneous velocity evolution (thin lines) are low-pass filtered (thick lines) to highlight trends due to the presence of the flame as opposed to turbulent fluctuations.

We first discuss the velocity evolution at the measurement point close to the center body wall (Fig. 13(b)). As the flame tongue swirls through the laser plane the approach flow is decelerated starting at time $t_{2}$ and subsequently recovers again starting around time $t_{5}$ indicating that the presumed elevated pressure causing the flow deflection is limited to the portion of the annulus occupied by a flame tongue. Interestingly, the cusp between the leading flame tongue and a secondary flame tongue is associated with the immediate spike in axial velocity at time $t_{4}$. The flame tongue furthermore accelerates the azimuthal velocity on its leading side (time $t_{2}$ ) and decelerates it on its trailing side (time $t_{6}$ ). Interestingly, the same acceleration and deceleration is observed rather far downstream of the leading flame tip. At time $t_{7}$, immediately before the flame front arrives, the azimuthal velocity is high. In contrast, the azimuthal velocity is low between the time the trailing side of the flame tongue passes through and $t_{9}$. These observations suggest that the presumed region of elevated pressure is not limited to the immediate vicinity of the leading flame tip. The described velocity pattern leads to predominantly swirling motion of the near-wall fluid ahead of the flame tongue (swirl angle of about $90^{\circ}$ between times $t_{2}$ and $t_{5}$ ) and a primarily axial fluid motion on the opposite side of the flame tongue. The low swirl and high axial velocity around times $t_{6}$ and $t_{9}$ indicates a reliving effect away from the flame tongue.

The second measurement point is close to the mixing tube wall and outside the burnt gas for all times show in Fig. 13(c). The decrease in azimuthal velocity starting around times $t_{5}$ and $t_{8}$ indicate the blockage effect of the flame tongue affects the flow close to the center body wall but also the incoming swirl flow beyond the radial portion occupied by burnt gas. The steady increase in axial velocity relative to the mean velocity (dashed line) close to the outer wall as the flame tongue swirls upstream further indicates an additional relieving affect for the unburnt gas. It appears that the path of least resistance for the flame to flashback is not to block the flow in the entire annulus or even transiently back-pressurize the entire flow, but only locally ahead of flame tongues.

A recent study was able to measure a static pressure rise on the center body wall inside the burnt gas region of a flame tongue swirling past the measurement port [38]. Our velocity field measurements further suggest that this increase in pressure is not limited to a region close to the center 
body wall and in addition has a magnitude capable of causing a time-rate-of-change in momentum associated with the changes in the velocity field just discussed.

\subsection{Differences and similarities of propagation modes}

Evidence for the existence of two different modes of upstream flame propagation along a wall during swirl flame boundary layer flashback has been presented. We now want to summarize the important features associated with each mode of flame propagation and discuss similarities as well as differences. The first mode consists of large-scale flame tongues swirling in the bulk flow direction as they propagate upstream along the center body wall as discussed in Section 3.4. and summarized in Fig. 14(a). This form of flame propagation is found to lead swirl-flame boundary-layer flashback under the conditions investigated in our work. The second mode consists of small-scale bulges aligned with the streamwise direction, which may counter-propagate into the approach flow as described in Section 3.3. and summarized in Fig. 14(b).

Boundary layer flashback in non-swirling channel flows has been found to be driven by smallscale bulges, which are correlated with the arrival of low-momentum streaks in the turbulent boundary layer $[16,17]$. Interestingly, we find that the formation of small-scale bulges in our swirl flow is only observed on the trailing side of the larger flame tongues where the flame surface is oriented perpendicular to the streamwise flow direction and hence provides the same alignment as in a $2 \mathrm{D}$ flow. The formation on the trailing side is likely assisted by the overall deceleration of the flow in that region due to the flame tongue itself. The formation, counter-propagation, break-up and downstream convection of small-scale bulges leads to a net upstream propagation of the flame brush in the negative streamwise direction in a channel flashback [16], which is not the case for the conditions investigated in our work as described in Sections 3.2. and 3.3. In contrast, the large flame tongues leading flashback in our model swirl combustor do not depend on the arrival of low-momentum fluid, which leads to a continuous, rather than intermittent, upstream propagation of the leading flame tip. The bulges may nonetheless contribute to flashback by additionally modifying the approach flow in the vicinity of the flame tip at occasions when they are formed at the tip of the flame tongue. Furthermore, they may modify the rate at which reactants are consumed by the flame tongue by increasing the flame surface area. Whether the consumption rate and hence the rate at which low density burnt gas is generated (generation of volume) is a dominant affect for the flashback mechanism requires further investigations, however.

Both modes of flame propagation have in common that they are coupled with the velocity field through the pressure field. Both propagation modes lead to a region of negative axial velocity ahead of the leading flame tip and hence enable flashback by strongly modifying the approach flow. However, the region of negative axial velocity is the result of a different underlying velocity field. In the case of 
flame tongues, the approach flow is deflected in the negative axial direction as indicated by the streamlines in Fig. 14(a) such that a predominantly swirling fluid motion with a small negative axial component exists upstream of the flame tip. No stagnation point exists upstream of the preheat zone in this case. As discussed in Section 3.4.3., the velocity field is modified far upstream and downstream of the leading flame tip as well as beyond the burnt gas region in the radial direction, which suggests that a large portion of the burnt gas is associated with elevated pressure as indicated by the grey area in Fig. 14(a). In contrast, the region of negative axial velocity upstream of small-scale bulges is the result of flow reversal in a small pocket ahead of the convex shaped flame tip with a stagnation point at its upstream end as sketched in Fig. 14(b). The DNS has shown that the elevated pressure is restricted to the immediate vicinity of the tip of convex bulges and subsequently decreases in the burnt gas [17], which is indicated by the grey spot in Fig. 14(b).

The newly found velocity field associated with flame tongues causing the observed flame propagation during swirl-flame flashback is summarized in more detail based on two locations along the flame front, positions 1 and 2, in Fig. 14(a). The azimuthal velocity is decreased on the trailing side of the flame tongue corresponding to a decrease in azimuthal momentum of the approach flow as indicated by the red arrow at position 1 . In contrast, the azimuthal velocity is increased towards the leading side (position 2) suggesting the flame tongue is pushing the flow ahead of itself. The red arrows are drawn to scale based on the velocity field measurement. The axial velocity along the leading side is found to be negative or at least significantly decelerated even far downstream of the flame tip. The displacement speed associated with the flame front is indicated by blue arrows assuming a flame speed of $0.7 \mathrm{~m} / \mathrm{s}$. The vector sum of local flow velocity and flame speed is indicated by the green arrows providing the propagation direction and speed of a local flame front element. The discrepancy in the azimuthal flame front propagation velocity between position 1 and 2 leads to the characteristic spreading of the flame tongue in the azimuthal direction with downstream distance. The positive axial component on the trailing side indicates that flame front elements are convected downstream in agreement with the high-speed chemiluminescence movies discussed in Section 3.2, which show structures along this side of the flame tongue being convected downstream by the flow. In contrast, the leading side of the flame tongue continuously propagates into a region of negative axial velocity that it created by pushing the flow ahead of itself.

\section{Conclusion}

Boundary layer flashback of swirl flames has been investigated experimentally in a model swirl combustor featuring a mixing tube with a single axial swirler and attached center body. Turbulent lean-premixed methane-air ( $\phi=0.8$ and 1.0) and hydrogen-methane-air flames $\left(95 \% \mathrm{H}_{2}\right.$ by vol., $\phi=0.4$ ) are investigated at atmospheric pressure and moderate Reynolds numbers up to 
$\mathrm{Re}_{\mathrm{h}}=8,000$. This work focuses on the upstream flame propagation during flashback events and the coupling between flame and approach flow in particular. A key component of our work is the timeresolved measurement of the velocity field in the vicinity of the leading flame tips during the upstream flame propagation both in planar slices (stereoscopic PIV) and in a volume (tomographic PIV). We demonstrate that an unambiguous interpretation of detected planar flame fronts and planar threecomponent velocity fields is possible in our configuration if combined with high-speed chemiluminescence imaging from a perpendicular point-of-view.

We show that small-scale bulges, similar to those that facilitate flashback in non-swirling channel flows, exist in boundary layer flashback in the current swirl flow. The formation and break-up process described by Eichler et al. [16] together with their finding of reverse flow pockets as well as the agreement of the measured three-dimensional velocity field with the DNS results by Gruber et al. [17] provide evidence that indeed the flame bulges observed in our swirl flow result from the same mechanism. We further show, however, that the formation, upstream propagation, break-up and downstream convection of these bulges on the trailing side of larger flame tongues do not lead to a net upstream flame propagation of the flame.

Instead, flashback is led by larger-scale flame tongues swirling with the bulk flow in the azimuthal direction as they propagate in the negative axial direction along the center body wall. We show that these large-scale flame tongues modify the approach flow differently. A deflection of streamlines ahead and around the flame tip causes a predominantly swirling motion including a region of negative axial velocity for the leading side of the flame tongue to propagate into. This region of negative axial velocity does not correspond to boundary layer separation or a recirculation bubble, and does not feature a stagnation point upstream of the preheat zone, as has been shown in channel-flow flashback $[16,17]$.

The formation of the observed flame tongues affects the flow on a larger scale compared to the small-scale bulges. This observation suggests that the presumed elevated pressure region causing the streamline deflection extends well into the burnt gas. Recent measurement of the wall pressure inside the burnt gas support this finding [38]. However, the region of negative axial velocity, which facilitates the upstream propagation, is still local as it only occupies the portion of the annulus in the vicinity of the flame tongue.

The investigated ultra-lean high-hydrogen content flames differ from the methane flames as they are significantly more convoluted. Despite a matched laminar flame speed, small-scale bulges form and resist the approach flow more frequently. Yet during flashback events these investigated hydrogen flames are found to still form larger tongues, which propagate upstream in a swirling motion with the smaller bulges being a superimposed perturbation. 
Real gas turbine combustors operate at high Reynolds numbers and elevated inlet temperatures and hence in a different regime of turbulent premixed combustion compared to the conditions studied in the current work. Further studies are needed to investigate whether the observations made here remain valid under real engine conditions.

\section{Acknowledgement}

This work was sponsored by the U.S. Department of Energy under grant DEFC2611-FE0007107. 


\section{References}

[1] B. Lewis, G. von Elbe, Stability and structure of burner flames, J. Chem. Phys. 11 (1943) 75-97.

[2] G. von Elbe, M. Mentser, Further Studies of the Structure and Stability of Burner Flames, J. Chem. Phys. 13 (1945) 89.

[3] K. Wohl, Quenching, Flash-Back, Blow-Off - Theory and Experiment, Symp. (Int.) Combust. 4 (1953) 68-89.

[4] L.E. Bollinger, R. Edse, Effect of Burner-Tip Temperature on Flash Back of Turbulent HydrogenOxygen Flames, Ind. Eng. Chem. 48 (1956) 802-807.

[5] B. Fine, Effect of Initial Temperature on Flash Back of Laminar and Turbulent Burner Flames, Ind. Eng. Chem. 51 (1958) 564-566.

[6] L.N. Khitrin, P.B. Moin, D.B. Smirnov, V.U. Shevchuk, Peculiarities of laminar- and turbulent-flame flashbacks, Symp. (Int.) Combust. 10 (1965) 1285-1291.

[7] S.L. Plee, A.M. Mellor, Review of Flashback Reported in Prevaporizing/Premixing Combustors, Combust. Flame. 32 (1978) 193-203.

[8] C. Eichler, G. Baumgartner, T. Sattelmayer, Experimental Investigation of Turbulent Boundary Layer Flashback Limits for Premixed Hydrogen-Air Flames Confined in Ducts, J. Eng. Gas Turbines Power. 134 (2012) 011502.

[9] B. Shaffer, Z. Duan, V. McDonell, Study of Fuel Composition Effects on Flashback Using a Confined Jet Flame Burner, J. Eng. Gas Turbines Power. 135 (2012) 011502.

[10] Z. Duan, B. Shaffer, V. McDonell, G. Baumgartner, T. Sattelmayer, Influence of Burner Material, Tip Temperature, and Geometrical Flame Configuration on Flashback Propensity of H 2 -Air Jet Flames, J. Eng. Gas Turbines Power. 136 (2013) 021502.

[11] Z. Duan, B. Shaffer, V. McDonell, Study of Fuel Composition, Burner Material, and Tip Temperature Effects on Flashback of Enclosed Jet Flame., J. Eng. Gas Turbines Power. 135 (2013) 121504.

[12] C. Eichler, T. Sattelmayer, Experiments on Flame Flashback in a Quasi-2D Turbulent Wall Boundary Layer for Premixed Methane-Hydrogen-Air Mixtures, J. Eng. Gas Turbines Power. 133 (2011) 011503.

[13] Y.-C. Lin, S. Daniele, P. Jansohn, K. Boulouchos, Turbulent Flame Speed as an Indicator for Flashback Propensity of Hydrogen-Rich Fuel Gases, J. Eng. Gas Turbines Power. 135 (2013) 111503.

[14] M. Konle, F. Kiesewetter, T. Sattelmayer, Simultaneous high repetition rate PIV-LIF-measurements of CIVB driven flashback, Exp. Fluids. 44 (2008) 529-538.

[15] C. Heeger, R.L. Gordon, M.J. Tummers, T. Sattelmayer, A. Dreizler, Experimental analysis of flashback in lean premixed swirling flames: upstream flame propagation, Exp. Fluids. 49 (2010) 853-863.

[16] C. Eichler, T. Sattelmayer, Premixed flame flashback in wall boundary layers studied by long-distance micro-PIV, Exp. Fluids. 52 (2011) 347-360.

[17] A. Gruber, J.H. Chen, D. Valiev, C.K. Law, Direct numerical simulation of premixed flame boundary layer flashback in turbulent channel flow, J. Fluid Mech. 709 (2012) 516-542.

[18] V. Kurdyumov, E. Fernández-Tarrazo, J.-M. Truffaut, J. Quinard, A. Wangher, G. Searby, Experimental and numerical study of premixed flame flashback, Proc. Combust. Inst. 31 (2007) 1275-1282.

[19] M. Konle, T. Sattelmayer, Interaction of heat release and vortex breakdown during flame flashback 
driven by combustion induced vortex breakdown, Exp. Fluids. 47 (2009) 627-635.

[20] A. Gruber, A.R. Kerstein, D. Valiev, C.K. Law, H. Kolla, J.H. Chen, Modeling of mean flame shape during premixed flame flashback in turbulent boundary layers, Proc. Combust. Inst. 35 (2015) 14851492.

[21] Y. Huang, V. Yang, Dynamics and stability of lean-premixed swirl-stabilized combustion, Prog. Energy Combust. Sci. 35 (2009) 293-364.

[22] T. Lieuwen, V. McDonell, D. Santavicca, T. Sattelmayer, Burner Development and Operability Issues Associated with Steady Flowing Syngas Fired Combustors, Combust. Sci. Technol. 180 (2008) 11691192.

[23] J. Fritz, M. Kröner, T. Sattelmayer, Flashback in a Swirl Burner With Cylindrical Premixing Zone, J. Eng. Gas Turbines Power. 126 (2004) 276.

[24] M. Kroener, T. Sattelmayer, J. Fritz, F. Kiesewetter, C. Hirsch, Flame Propagation in Swirling FlowsEffect of Local Extinction on the Combustion Induced Vortex Breakdown, Combust. Sci. Technol. 179 (2007) 1385-1416.

[25] Y. Sommerer, D. Galley, T. Poinsot, Large eddy simulation and experimental study of flashback and blow-off in a lean partially premixed swirled burner, J. Turbul. 5 (2004).

[26] S. Tanimura, M. Komiyama, K. Takeishi, Y. Iwasaki, K. Nakayama, Visualization of flashback in a premixed burner with swirling flow, Sci. China Ser. E Technol. Sci. 53 (2010) 40-45.

[27] G. Blesinger, R. Koch, H.-J. Bauer, Influence of flow field scaling on flashback of swirl flames, Exp. Therm. Fluid Sci. 34 (2010) 290-298.

[28] A. Schönborn, P. Sayad, J. Klingmann, Influence of precessing vortex core on flame flashback in swirling hydrogen flames, Int. J. Hydrogen Energy. 39 (2014) 20233-20241.

[29] E. Tangermann, M. Pfitzner, M. Konle, T. Sattelmayer, Large-Eddy Simulation and Experimental Observation of Combustion-Induced Vortex Breakdown, Combust. Sci. Technol. 182 (2010) 505-516.

[30] C. Mayer, J. Sangl, T. Sattelmayer, Study on the Operational Window of a Swirl Stabilized Syngas Burner Under Atmospheric and High Pressure Conditions, J. Eng. Gas Turbines Power. 134 (2012) 031506.

[31] S. Ishizuka, Flame propagation along a vortex axis, Prog. Energy Combust. Sci. 28 (2002) 477-542.

[32] W.T. Ashurst, Flame Propagation Along a Vortex: the Baroclinic Push, Combust. Sci. Technol. 112 (1996) 175-185.

[33] F. Kiesewetter, M. Konle, T. Sattelmayer, Analysis of Combustion Induced Vortex Breakdown Driven Flame Flashback in a Premix Burner With Cylindrical Mixing Zone, J. Eng. Gas Turbines Power. 129 (2007) 929-936.

[34] M. Konle, T. Sattelmayer, Time Scale Model for the Prediction of the Onset of Flame Flashback Driven by Combustion Induced Vortex Breakdown, J. Eng. Gas Turbines Power. 132 (2010) 041503.

[35] A. Nauert, P. Petersson, M. Linne, A. Dreizler, Experimental analysis of flashback in lean premixed swirling flames: conditions close to flashback, Exp. Fluids. 43 (2007) 89-100.

[36] B. Dam, G. Corona, M. Hayder, A. Choudhuri, Effects of syngas composition on combustion induced vortex breakdown (CIVB) flashback in a swirl stabilized combustor, Fuel. 90 (2011) 3274-3284.

[37] A. De, S. Acharya, Parametric study of upstream flame propagation in hydrogen-enriched premixed combustion: Effects of swirl, geometry and premixedness, Int. J. Hydrogen Energy. 37 (2012) 14649- 
14668.

[38] N. Karimi, C. Heeger, L. Christodoulou, A. Dreizler, Experimental and Theoretical Investigation of the Flashback of a Swirling, Bluff-Body Stabilised, Premixed Flame, Zeitschrift Für Phys. Chemie. 229 (2015) 663-689.

[39] T. Sattelmayer, C. Mayer, J. Sangl, Interaction of Flame Flashback Mechanisms in Premixed HydrogenAir Swirl Flames, J. Eng. Gas Turbines Power. 138 (2014) 1-13.

[40] D. Ebi, N.T. Clemens, Simultaneous high-speed 3D flame front detection and tomographic PIV, Meas. Sci. Technol. 27 (2016) 035303.

[41] L. Boyer, Laser Tomographic Method for Flame Front Movement Studies, Combust. Flame. 39 (1980) 321-323.

[42] P.C. Miles, F.C. Gouldin, Simultaneous measurements of flamelet position and gas velocity in premixed turbulent flames, in: Proc. 1987 ASME-JSME Therm. Eng. Jt. Conf., 1987: pp. 187-193.

[43] T. Chew, R. Britter, K. Bray, Laser tomography of turbulent premixed bunsen flames, Combust. Flame. 75 (1989) 165-174.

[44] P.C. Miles, F.C. Gouldin, Premixed Turbulent Flame/Flow Interaction: Simultaneous Measurements of Velocity and Flamelet Position, AIAA J. 36 (1998) 1178-1189.

[45] I. Shepherd, E. Bourguignon, Y. Michou, I. Gökalp, The burning rate in turbulent Bunsen flames, Symp. (Int.) Combust. 27 (1998) 909-916.

[46] Ö.L. Gülder, G.J. Smallwood, R. Wong, D.R. Snelling, R. Smith, B.M. Descamps, et al., Flame front surface characteristics in turbulent premixed propane air combustion, Combust. Flame. 120 (2000) 407416.

[47] B. Renou, A. Boukhalfa, D. Puechberty, M. Trinite, Local scalar flame properties of freely propagating premixed turbulent flames at various Lewis numbers, Combust. Flame. 123 (2000) 507-521.

[48] D.A. Knaus, F.C. Gouldin, Measurements of flamelet orientations in premixed flames with positive and negative markstein numbers, Proc. Combust. Inst. 28 (2000) 367-373.

[49] S. Balusamy, A. Cessou, B. Lecordier, Direct measurement of local instantaneous laminar burning velocity by a new PIV algorithm, Exp. Fluids. 50 (2010) 1109-1121.

[50] J. Kerl, C. Lawn, F. Beyrau, Three-dimensional flame displacement speed and flame front curvature measurements using quad-plane PIV, Combust. Flame. 160 (2013) 2757-2769.

[51] A. Marshall, J. Lundrigan, P. Venkateswaran, J. Seitzman, T. Lieuwen, Fuel effects on leading point curvature statistics of high hydrogen content fuels, Proc. Combust. Inst. 35 (2015) 1417-1424.

[52] B. Wieneke, PIV uncertainty quantification from correlation statistics, Meas. Sci. Technol. 26 (2015) 074002.

[53] F. Scarano, Tomographic PIV: principles and practice, Meas. Sci. Technol. 24 (2013) 012001.

[54] B. Wieneke, Volume self-calibration for 3D particle image velocimetry, Exp. Fluids. 45 (2008) 549-556.

[55] M. Novara, K.J. Batenburg, F. Scarano, Motion tracking-enhanced MART for tomographic PIV, Meas. Sci. Technol. 21 (2010) 035401.

[56] K.P. Lynch, F. Scarano, Experimental determination of tomographic PIV accuracy by a 12-camera system, Meas. Sci. Technol. 25 (2014) 084003. 
[57] J. Westerweel, F. Scarano, Universal outlier detection for PIV data, Exp. Fluids. 39 (2005) 1096-1100.

[58] G.E. Elsinga, C. Poelma, A. Schröder, R. Geisler, F. Scarano, J. Westerweel, Tracking of vortices in a turbulent boundary layer, J. Fluid Mech. 697 (2012) 273-295.

[59] C.K. Law, C. Sung, Structure, aerodynamics, and geometry of premixed flamelets, Prog. Energy Combust. Sci. 26 (2000) 459-505. 


\section{Figure captions for manuscript CNF-D-15-00744}

Fig. 1: Model swirl combustor.

Fig. 2: Planar Laser Mie Scattering setup providing the three-component velocity field and flame front location in an axial-radial plane inside the mixing tube.

Fig. 3: Dewarped particle image with flame (black region corresponds to burnt gas) and extracted planar flame front shown in red.

Fig. 4: Diagnostic setup for the three-dimensional flame topography and volumetric velocity field measurement.

Fig. 5: Mean non-reacting radial velocity profiles $60 \mathrm{~mm}$ upstream of mixing tube axis. Circles denote $\mathrm{Re}_{\mathrm{h}}=4,000$ and pluses denote $\mathrm{Re}_{\mathrm{h}}=8,000$ case. Axial velocity (blue), azimuthal velocity (green), streamwise velocity (black) and local swirl angle (red) are shown.

Fig. 6: Chemiluminescence images at selected time steps of a $\mathrm{CH}_{4}$-air flame during flashback recorded at $2 \mathrm{kHz}$.

Fig. 7: Chemiluminescence image sequence showing the upstream flame propagation and flame structure of a $\mathrm{CH}_{4}$-air flame (a) and a $\mathrm{H}_{2} / \mathrm{CH}_{4}$-air flame (b) in the field-of-view marked in Fig. 6. Corresponding movies are Video 1(a) and Video 1(b).

Fig. 8: Formation of a small-scale flame bulge on the trailing side of a flame tongue (luminescence image sequence, top row). Axial velocity field in $y=0.8 \mathrm{~mm}$ plane and $3 \mathrm{D}$ streamlines showing (a) the reverse flow pocket associated with the flame bulge as it resists the approach flow and (b) the recovered flow field subsequently convecting the bulge downstream. Streamlines are colored by $y$-distance from the center body as explained in (c). The flame surface rendered in grey corresponds to an isotherm of about $555 \mathrm{~K}$.

Fig. 9: Simultaneous chemiluminescence (top row) and velocity field measurement (bottom row) highlighting the difference between apparent and true upstream flame propagation and its correlation with a region of negative axial velocity. The planar flame front (red line) and axial velocity field is shown in a radial-axial slice corresponding to the green line in the luminescence images. The white line in (d) is an isoline of $0 \mathrm{~m} / \mathrm{s}$ indicating the region of negative axial velocity.

Fig. 10: Velocity field and region of negative axial velocity (white line) in the vicinity of the leading flame front (red line) in a slice through the leading side of the flame tongue (a) and through the flame tip (b). 
Fig. 11: Three-dimensional velocity field associated with flame tongues (grey surface): (a) Axial velocity field in $y=0.8 \mathrm{~mm}$ plane and $3 \mathrm{D}$ streamlines. (b) Velocity field in $x=0$ plane. Shown here: $\mathrm{CH}_{4}$-air flame $(\phi=0.8)$.

Fig. 12: Azimuthal vorticity field in a radial-axial slice in the vicinity of a flame tongue (red line marks flame front) leading flashback. The black dashed line highlights a layer of vorticity moving coherently based on the movie sequence (see Video 2).

Fig. 13: Time traces of axial velocity (blue), azimuthal velocity (green) and swirl angle (red) at two radial locations (b) in the boundary layer close to the center body and (c) close to the mixing tube wall, respectively. Mean velocities are marked by horizontal dashed lines. The global flame position in relation to the measurement points (white cross, same axial location) is shown in (a) at nine instants in time.

Fig. 14: Schematics summarizing flame shape, flame propagation direction and important flow features associated with (a) flame tongues leading boundary layer flashback in swirl flows and (b) flame bulges counter-propagating into the approach flow on the trailing side of flame tongues. 


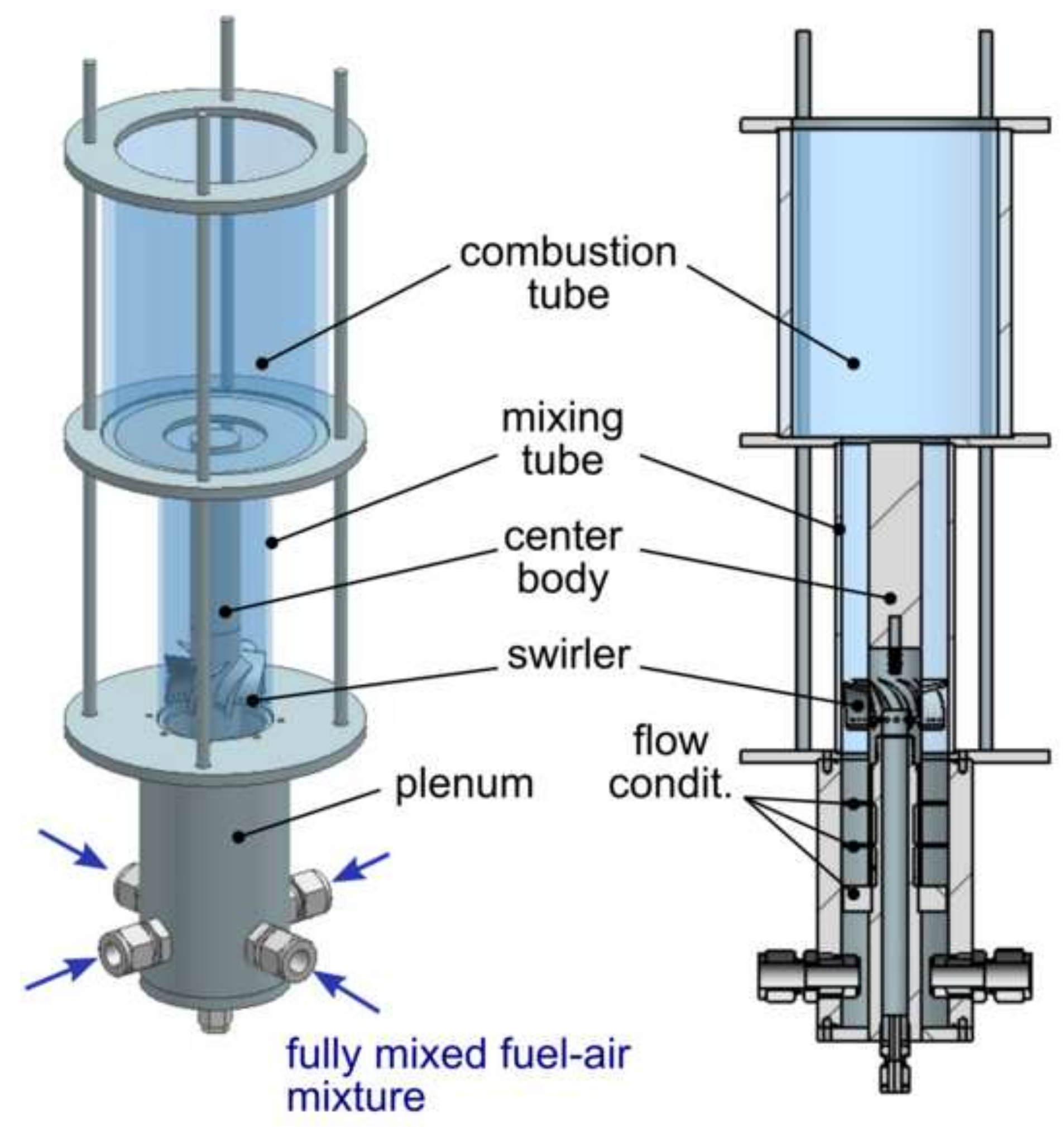

fully mixed fuel-air mixture 


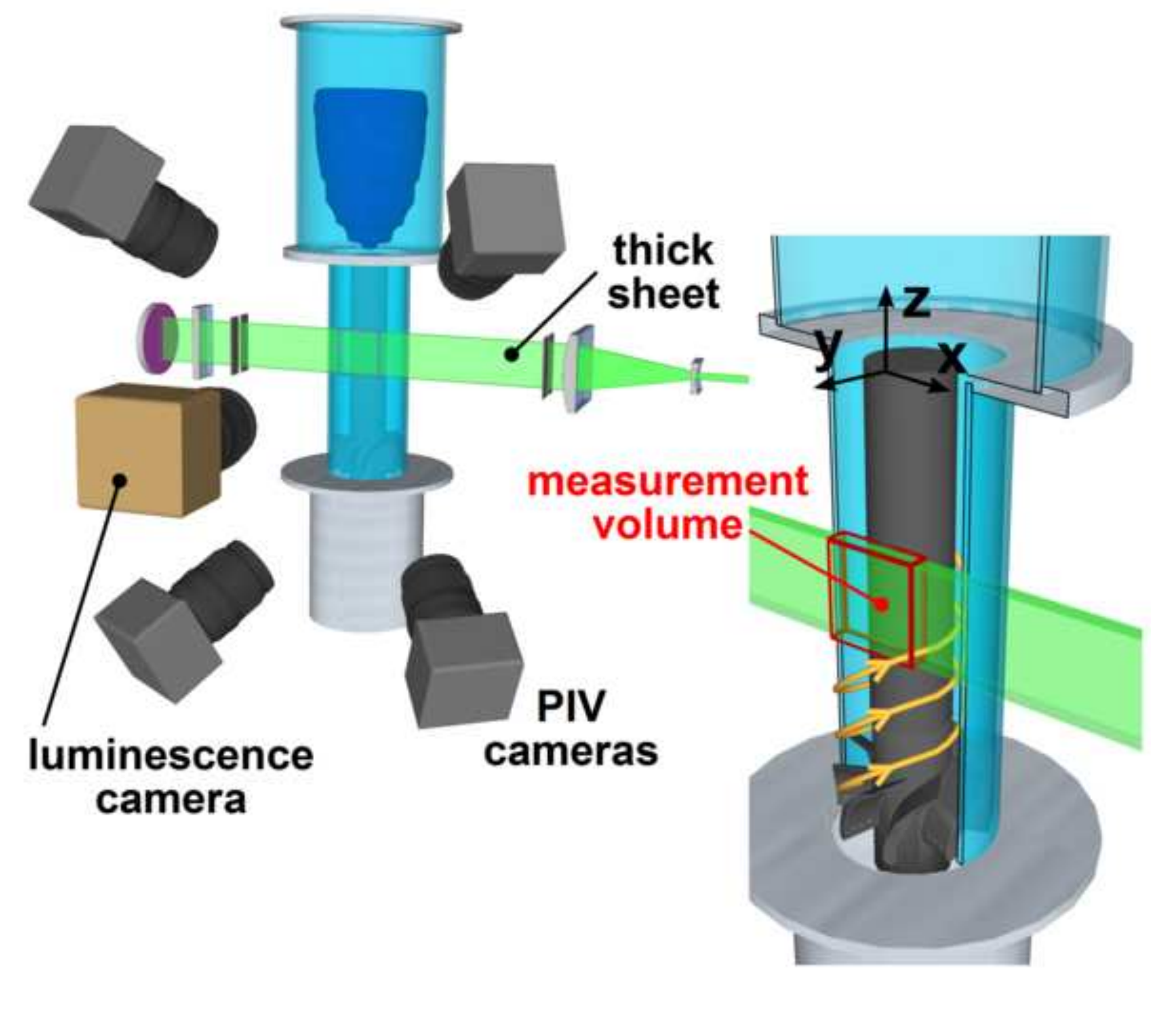
camera cameras
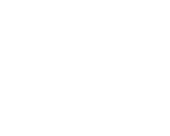


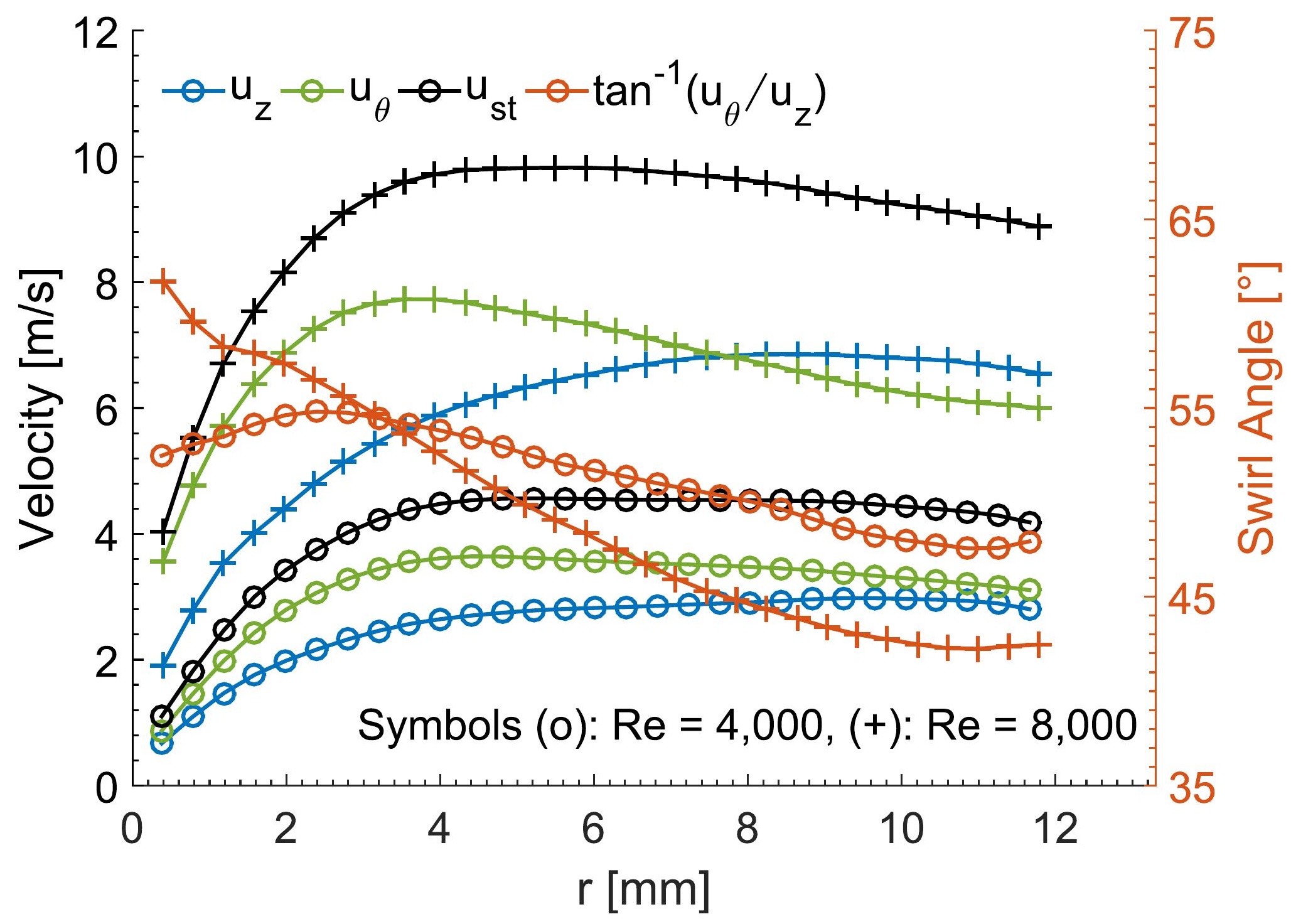



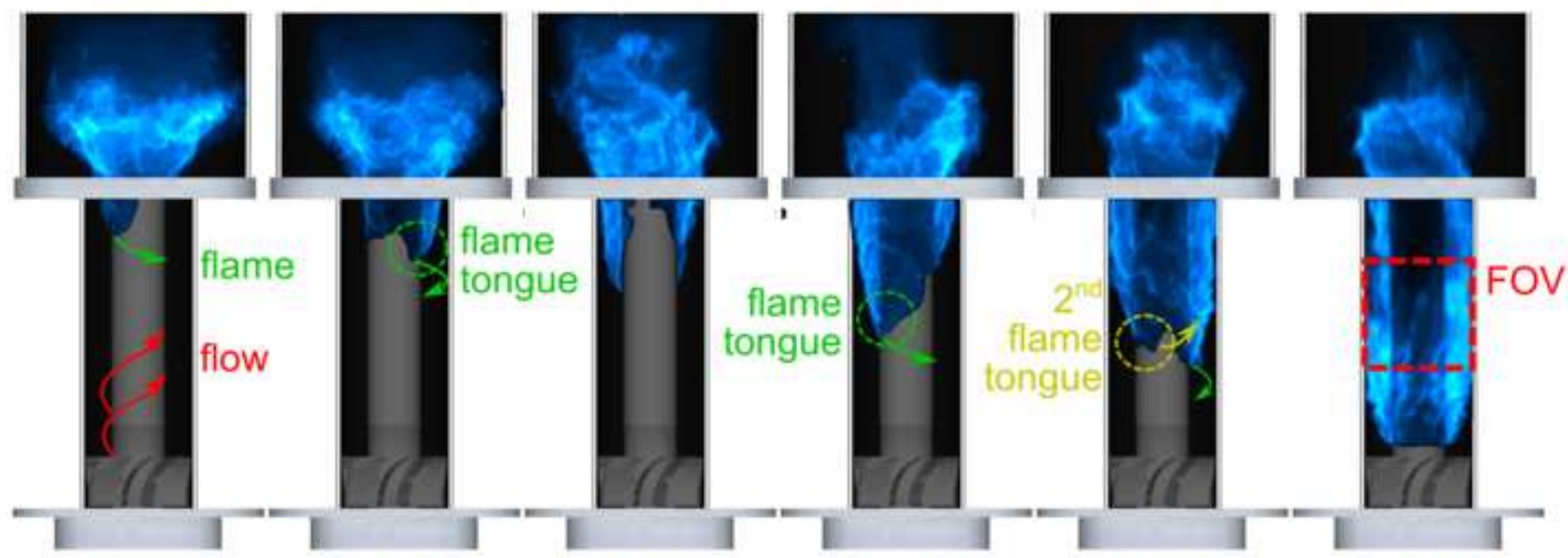

$\mathrm{t}_{0}+142 \mathrm{~ms}$

$\mathrm{t}_{0}+194 \mathrm{~ms}$

$\mathrm{t}_{0}+257 \mathrm{~ms}$

$\mathrm{t}_{0}+305 \mathrm{~ms}$

$\mathrm{t}_{0}+355 \mathrm{~ms}$

$\mathrm{t}_{0}+421 \mathrm{~ms}$ 

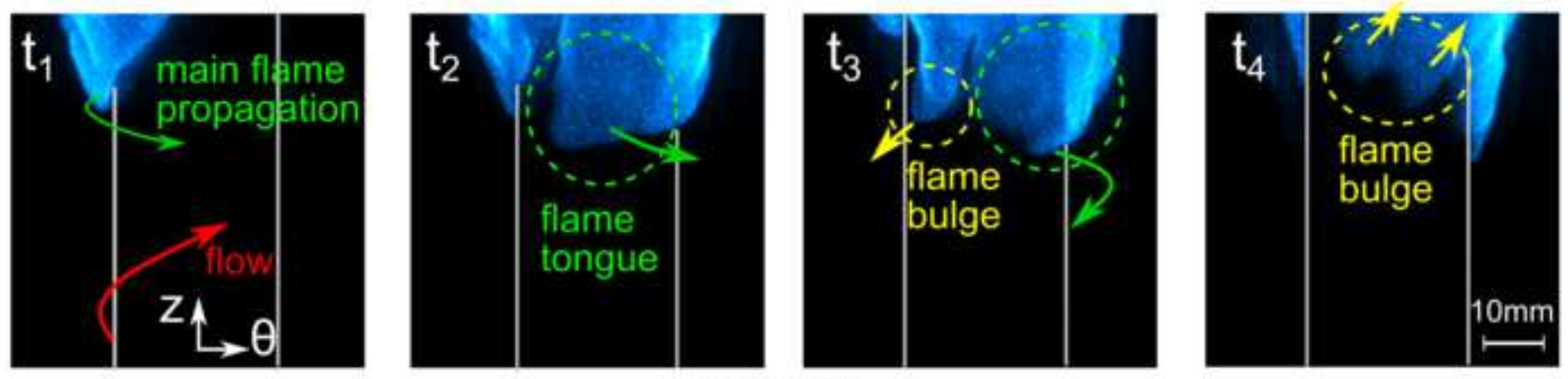

\section{(a) $\mathrm{CH}_{4}$}
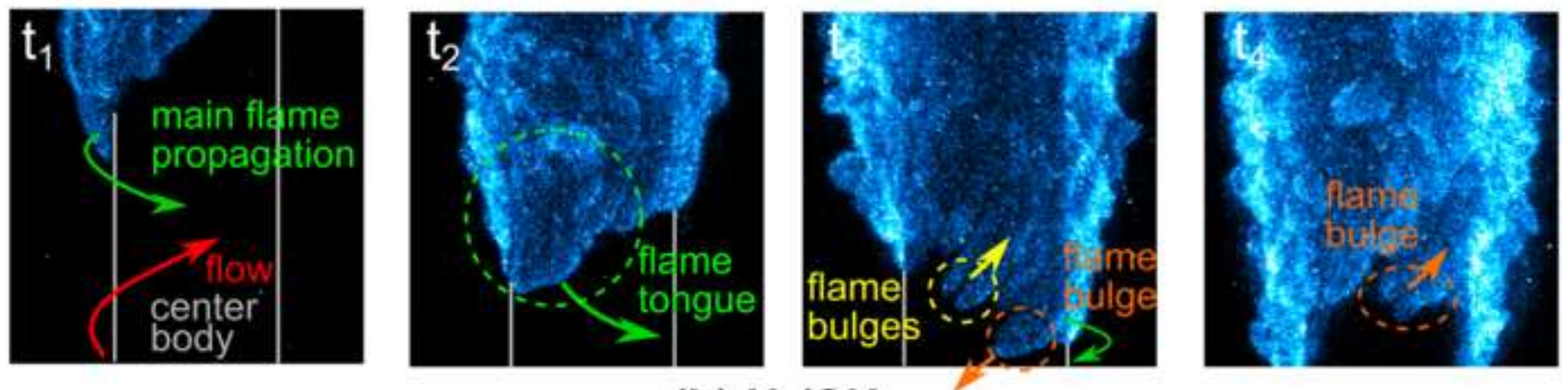

(b) $\mathrm{H}_{2} / \mathrm{CH}_{4}$ 


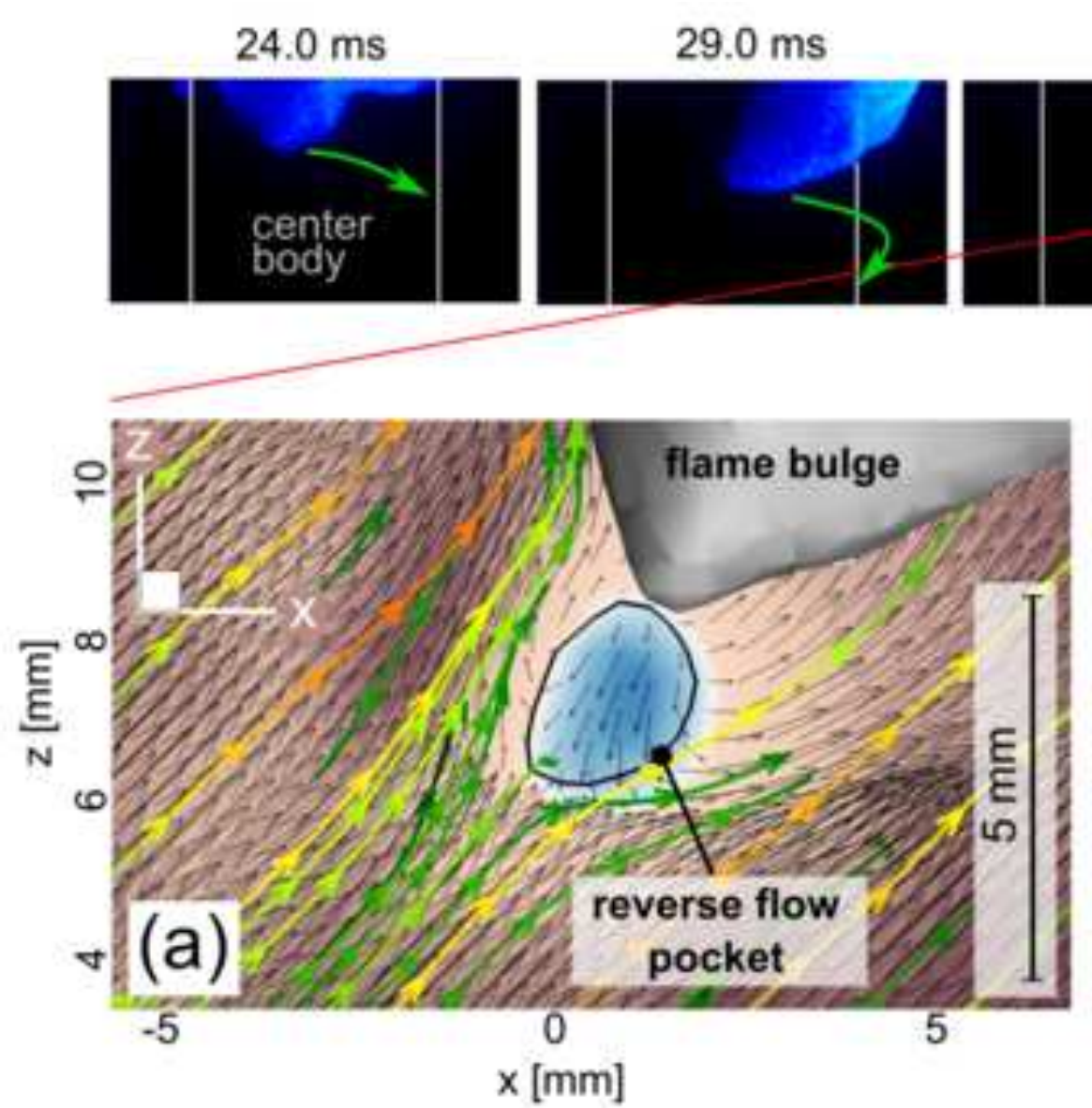

$31.0 \mathrm{~ms}$

$32.2 \mathrm{~ms}$

$34.0 \mathrm{~ms}$
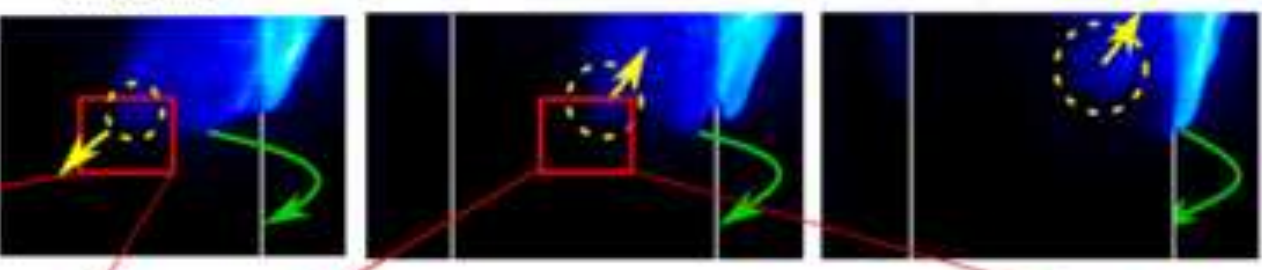

$>$

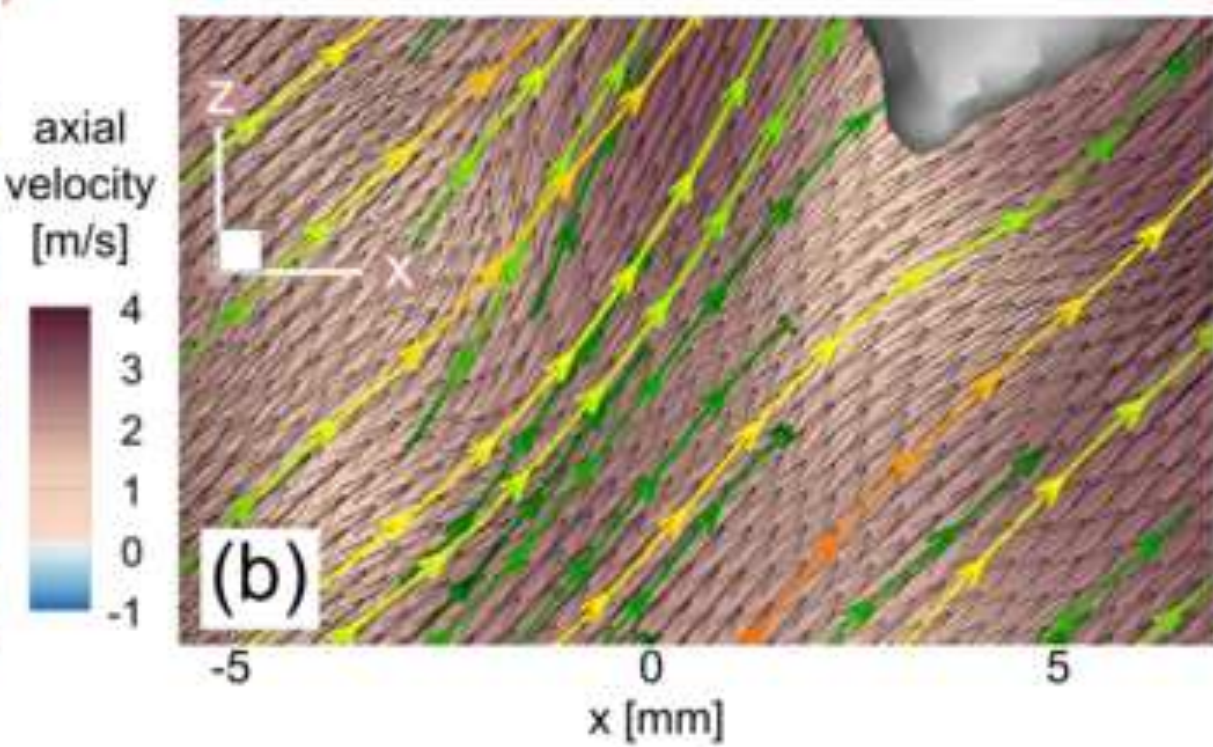

(c) bottom view

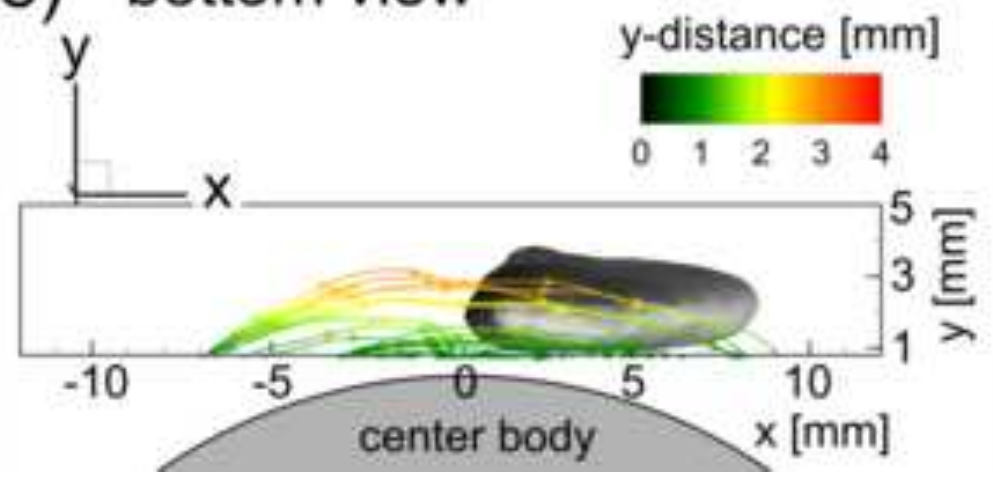



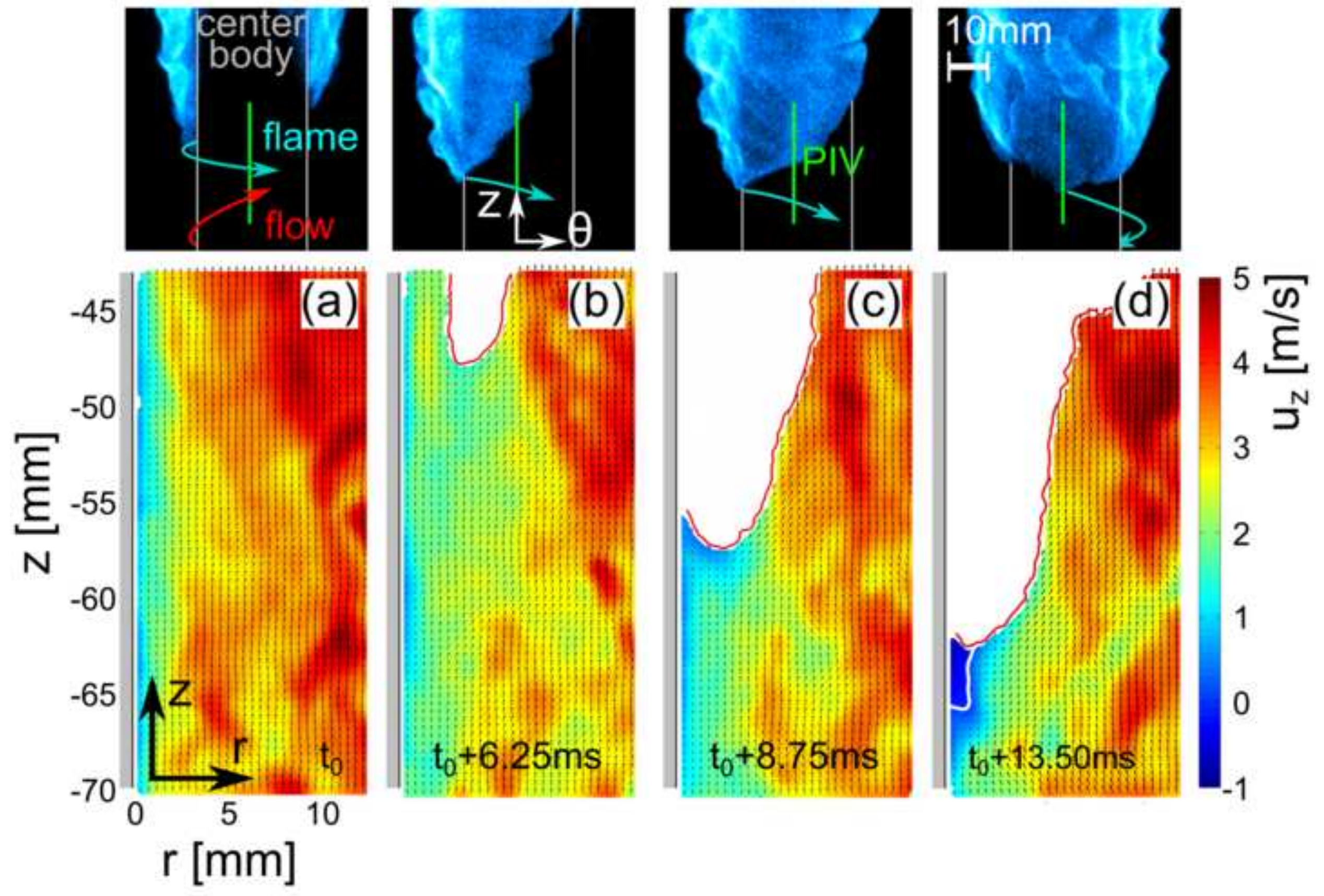

$r$ [mm] 

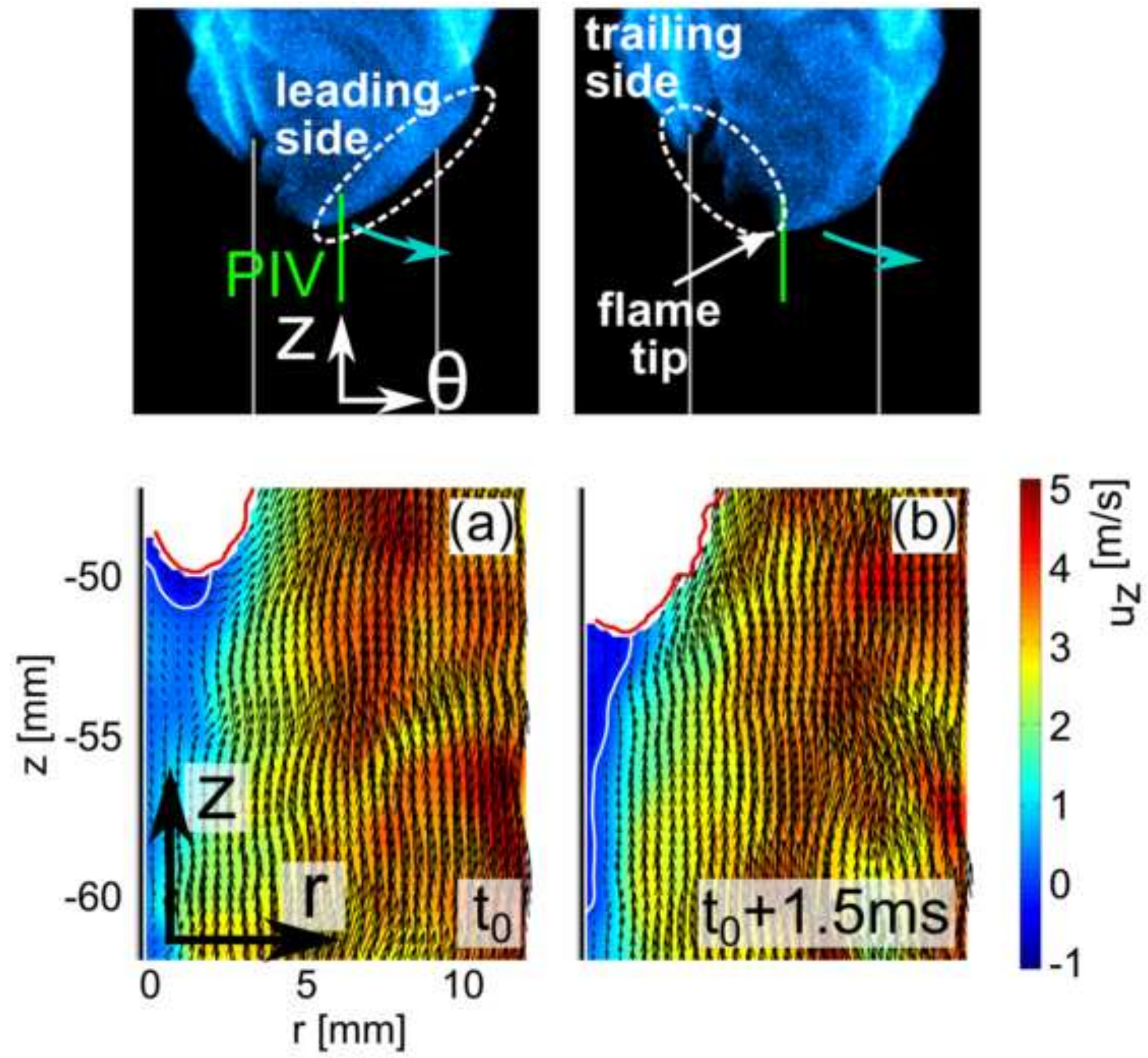

2

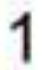

0

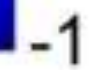

$$
\mathrm{r}[\mathrm{mm}]
$$



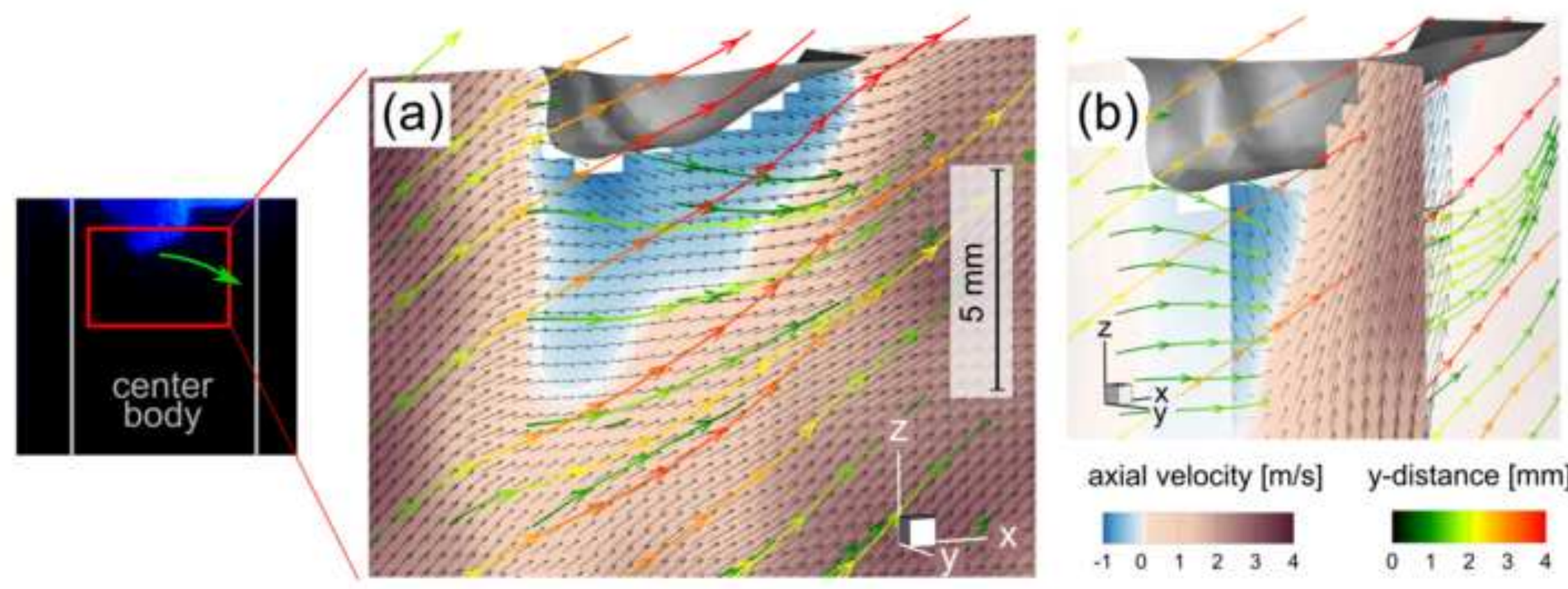

axial velocity $[\mathrm{m} / \mathrm{s}] \quad y$-distance $[\mathrm{mm}]$

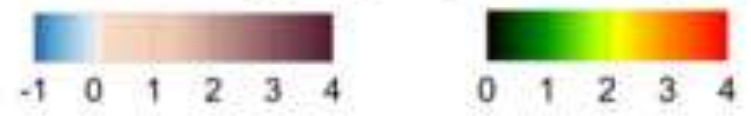

(b)

$\begin{array}{llllll}-1 & 0 & 1 & 2 & 3 & 4\end{array}$ 

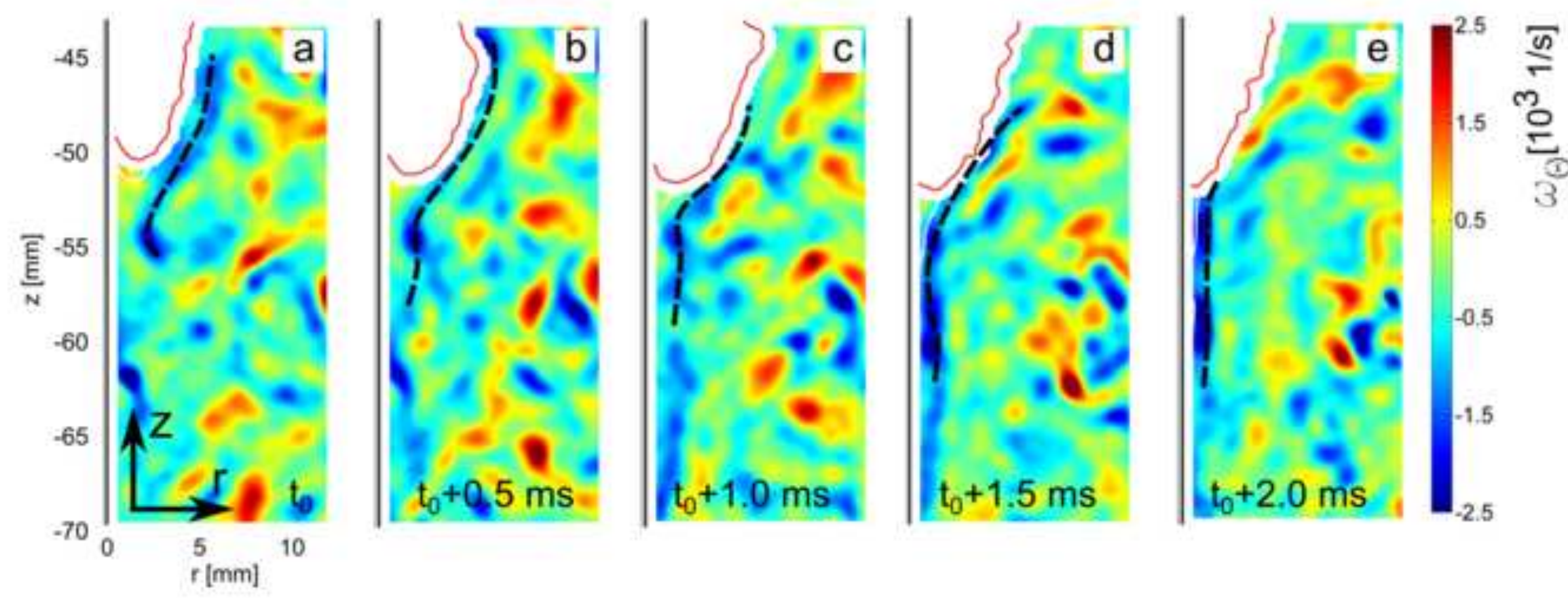

$\frac{5}{3}$

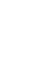

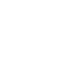




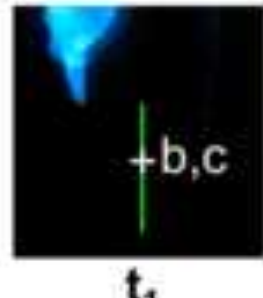

$t_{1}$

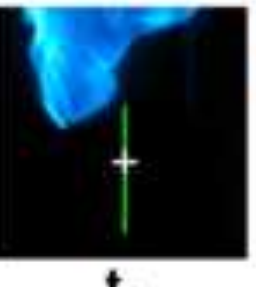

$t_{2}$

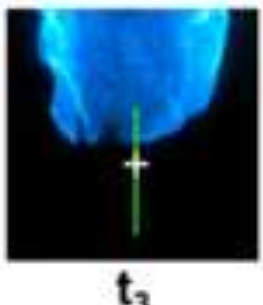

$t_{3}$

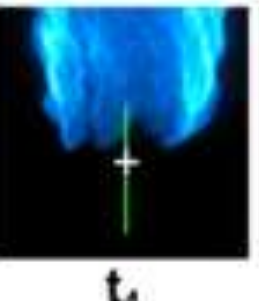

$t_{4}$

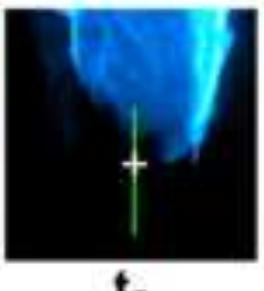

$t_{5}$

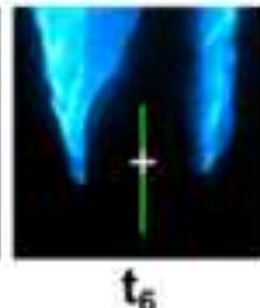

$t_{6}$

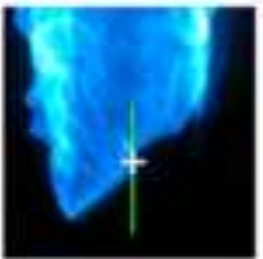

$\mathrm{t}_{7}$

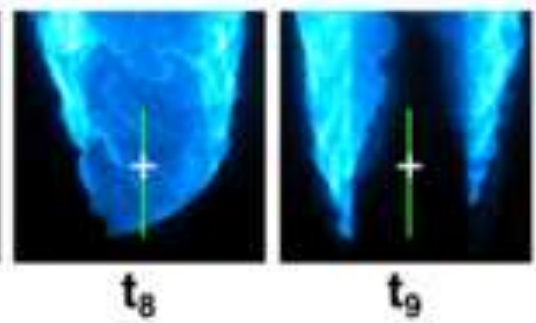

(a)

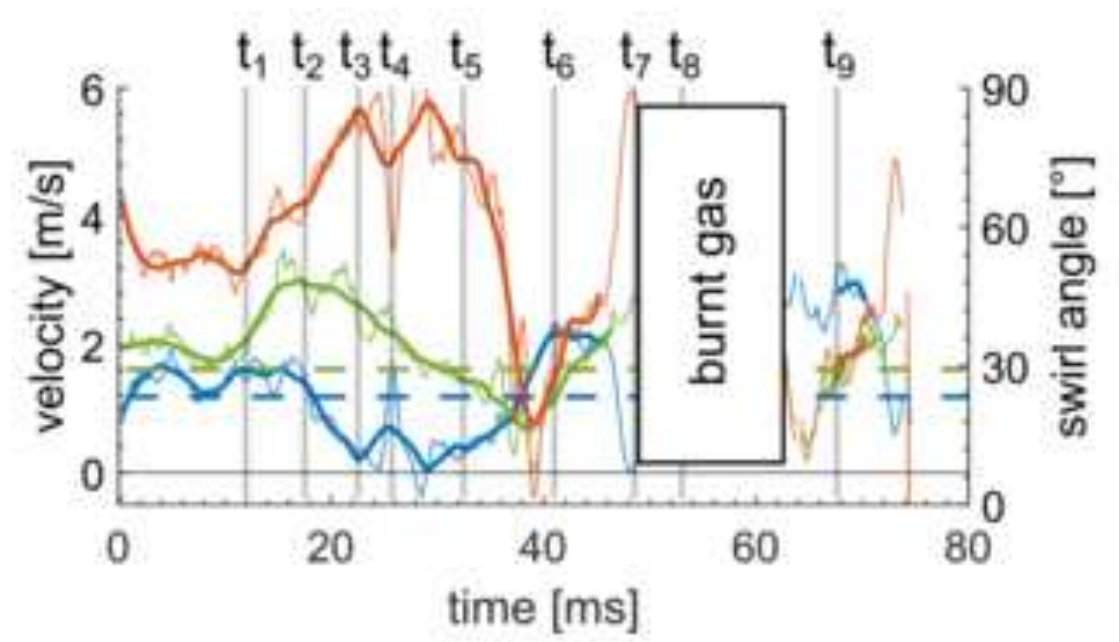

(b) $r=0.75 \mathrm{~mm}$

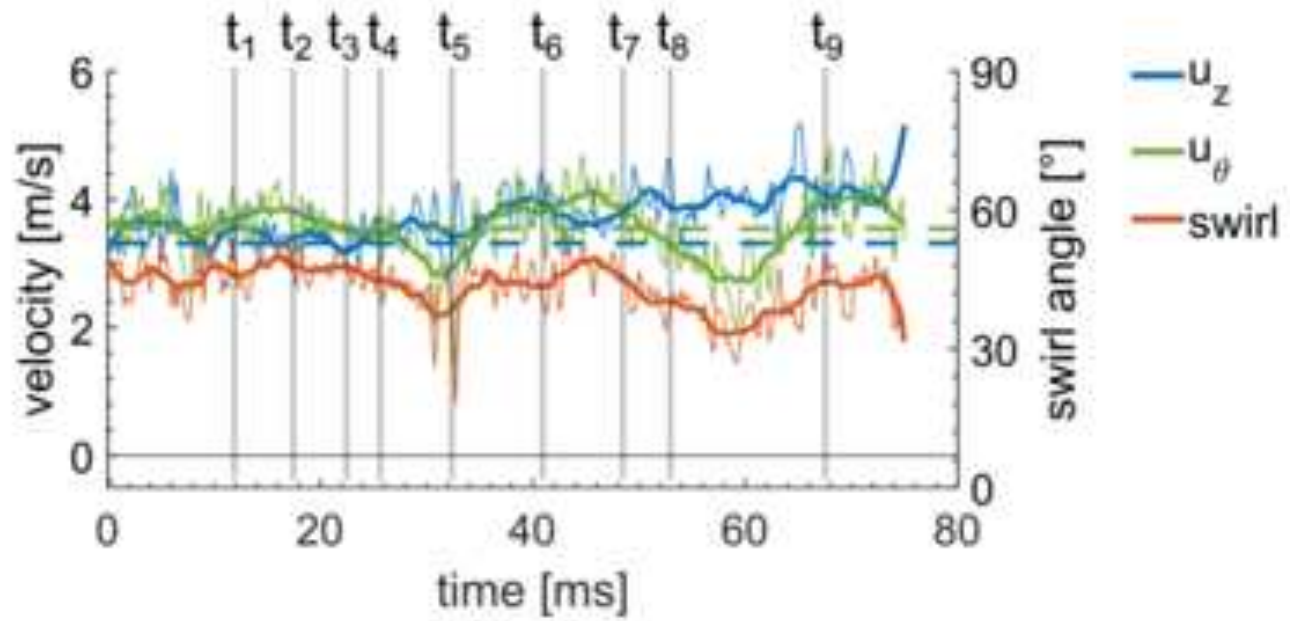

(c) $r=11.0 \mathrm{~mm}$ 


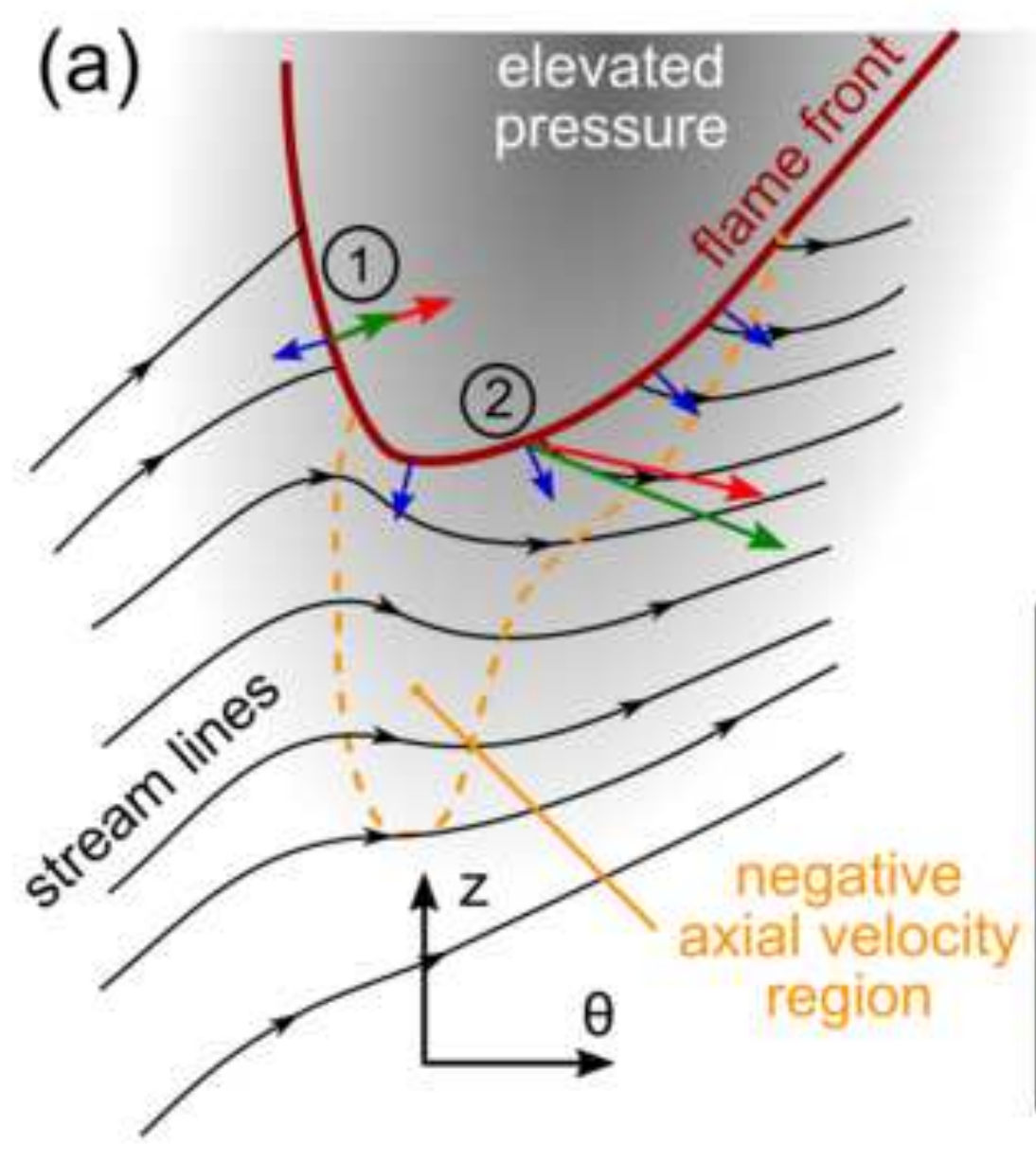

(b)

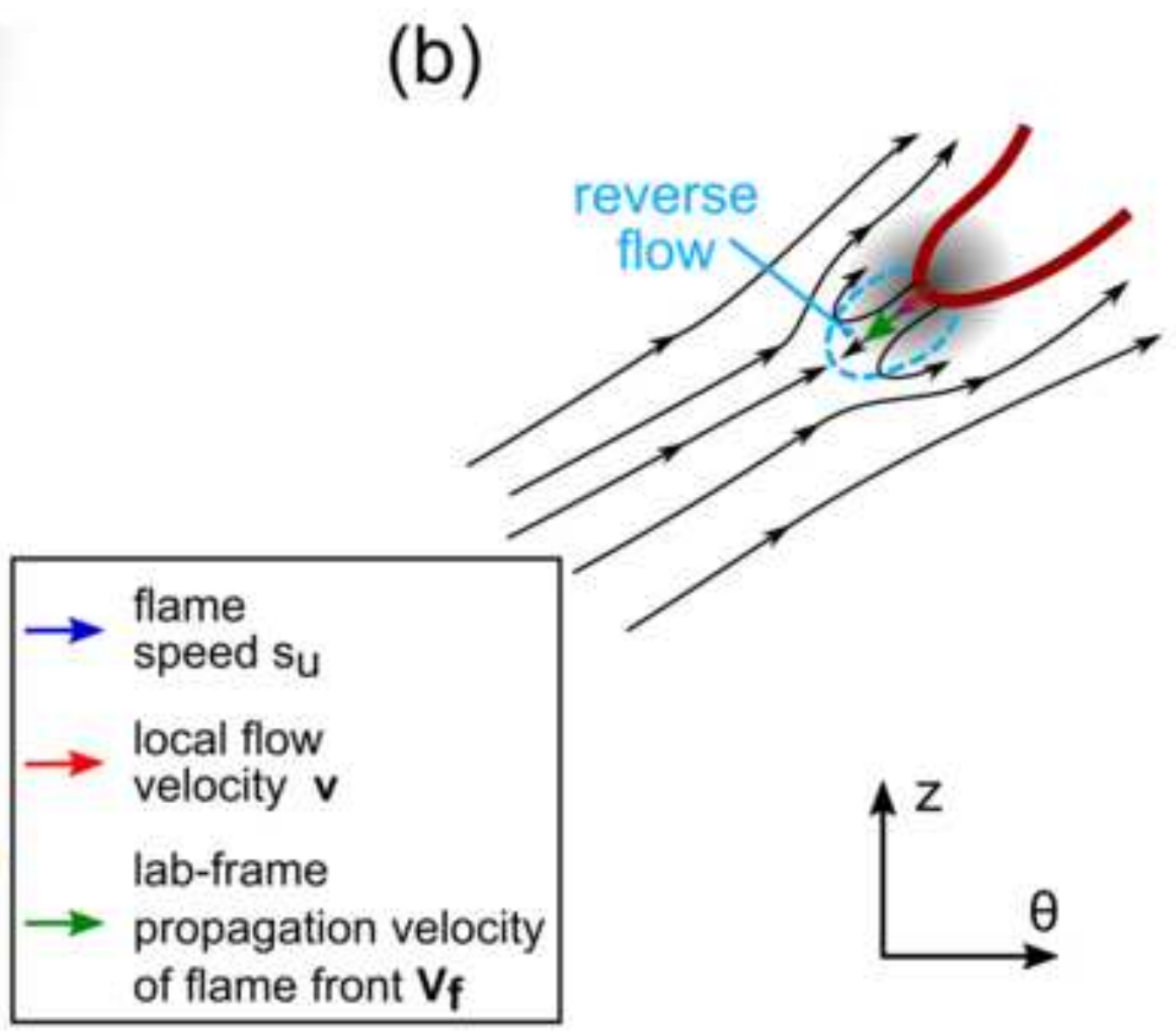

